\title{
The Forced van der Pol Equation II: Canards in the Reduced System*
}

\author{
Katherine Bold ${ }^{\dagger}$, Chantal Edwards ${ }^{\ddagger}$, John Guckenheimer ${ }^{\S}$, Sabyasachi Guharay , \\ Kathleen Hoffman ${ }^{\ddagger}$, Judith Hubbardll, Ricardo Oliva ${ }^{\S}$, and Warren Weckesser**
}

Abstract. This is the second in a series of papers about the dynamics of the forced van der Pol oscillator [J. Guckenheimer, K. Hoffman, and W. Weckesser, SIAM J. Appl. Dyn. Syst., 2 (2003), pp. 1-35]. The first paper described the reduced system, a two dimensional flow with jumps that reflect fast trajectory segments in this vector field with two time scales. This paper extends the reduced system to account for canards, trajectory segments that follow the unstable portion of the slow manifold in the forced van der Pol oscillator. This extension of the reduced system serves as a template for approximating the full nonwandering set of the forced van der Pol oscillator for large sets of parameter values, including parameters for which the system is chaotic. We analyze some bifurcations in the extension of the reduced system, building upon our previous work in [J. Guckenheimer, K. Hoffman, and W. Weckesser, SIAM J. Appl. Dyn. Syst., 2 (2003), pp. 1-35]. We conclude with computations of return maps and periodic orbits in the full three dimensional flow that are compared with the computations and analysis of the reduced system. These comparisons demonstrate numerically the validity of results we derive from the study of canards in the reduced system.

Key words. van der Pol oscillator, hybrid dynamical system, bifurcations, canards, chaotic invariant set

AMS subject classifications. $37 \mathrm{C} 10,37 \mathrm{C} 27,37 \mathrm{G} 15$

DOI. $10.1137 / \mathrm{S} 1111111102419130$

1. Introduction. We study the forced van der Pol oscillator [24] in the form

$$
\begin{aligned}
\varepsilon \dot{x} & =y+x-\frac{x^{3}}{3} \\
\dot{y} & =-x+a \sin (2 \pi \theta) \\
\dot{\theta} & =\omega
\end{aligned}
$$

where $\theta$ is a cyclic variable belonging to $\mathbb{R} / \mathbb{Z}$. The critical manifold of this system is the surface $C$ defined by $\dot{x}=0$; i.e., $y=x^{3} / 3-x$. We divide the critical manifold into $C_{s}=$ $\{(x, y, \theta) \in C|| x \mid>1\}, C_{u}=\{(x, y, \theta) \in C|| x \mid<1\}$, and the two fold curves $S_{ \pm 1}$, where

* Received by the editors December 3, 2002; accepted for publication (in revised form) by M. Golubitsky July 21, 2003; published electronically December 22, 2003. This research was partially supported by the National Science Foundation and the Department of Energy. The results were obtained during a Research Experiences for Undergraduates Program at Cornell University sponsored by the National Science Foundation.

http://www.siam.org/journals/siads/2-4/41913.html

${ }^{\dagger}$ Mathematics Department, University of Texas, Austin, TX 78712.

${ }^{\ddagger}$ Department of Mathematics and Statistics, University of Maryland, Baltimore County, Baltimore, MD 21250 (khoffman@math.umbc.edu).

${ }^{\S}$ Mathematics Department, Cornell University, Ithaca, NY 14853 (gucken@cam.cornell.edu, RAOliva@lbl.gov).

"Department of Operations Research and Financial Engineering, Princeton University, Princeton, NJ 08544.

"Geology Department, California Institute of Technology, Pasadena, CA 91125.

** Mathematics Department, Colgate University, Hamilton, NY 13346 (wweckesser@mail.colgate.edu). 
$S_{\xi}=\{(x, y, \theta) \in C \mid x=\xi\}$. The slow flow on the critical manifold solves the two dimensional system of differential equations derived from (1.1) by setting $\varepsilon=0$, differentiating $y=x^{3} / 3-x$ to obtain $\dot{y}=\left(x^{2}-1\right) \dot{x}$ on the critical manifold and rescaling time:

$$
\begin{aligned}
\theta^{\prime} & =\omega\left(x^{2}-1\right), \\
x^{\prime} & =-x+a \sin (2 \pi \theta) .
\end{aligned}
$$

The reduced system is given by augmenting the slow flow with the rules that trajectories jump from the fold curve $S_{1}$ to $S_{-2}$ and from the fold curve $S_{-1}$ to $S_{2}$, in each case preserving the value of $\theta$. The reduced system with its jumps is a hybrid dynamical system [2]. We define a half return map $H$ in terms of candidates [4, p. 68] and the symmetry operation $T(x, \theta)=(-x, \theta+0.5)$ of the slow flow. Specifically, $H: S_{2} \rightarrow S_{2}$ is defined by following the slow dynamics from $S_{2}$ to $S_{1}$, jumping with constant $\theta$ from $S_{1}$ to $S_{-2}$, and then applying the symmetry $T$. Details of these constructions can be found in [14], together with extensive analysis of properties of $H$. Additionally, in section 4, we formulate a half Poincaré map of the full system and a singular half Poincaré map of the reduced sytem to further clarify the relationship between solutions of the half return map and solutions of the full forced van der Pol equation.

"Fenichel theory" [10] and a theorem of Levinson [17] imply that the reduced system gives approximations to trajectories of (1.1) that have nondegenerate slow-fast decompositions [13]. These are trajectories that approach the fold curves $S_{ \pm 1}$ at regular points of the slow flow. When $|a|<1$, all points on the fold curves $S_{ \pm 1}$ are regular and $H$ is a diffeomorphism of the circle $S_{2}$. When $|a|>1$, there are four equilibria of the slow flow on the fold curves, called folded equilibria. A symmetric pair of the folded equilibria are always saddles. The properties of the system (1.1) are more complicated in the vicinity of the folded equilibria. Benoit [4] has studied the flow of slow-fast vector fields with two slow variables and one fast variable in the vicinity of folded equilibria. We review his results for folded saddles in the next section; they play an important role in our extension of the reduced system and its return map. The analysis of folded nodes is incomplete, and this leads to a gap in our description of the reduced system for small parameter ranges near $a=1$ where folded nodes exist.

There are trajectories of the three dimensional system (1.1) that cross the fold curve near the folded saddles and continue along the unstable portion of the slow manifold. The trajectory segments that follow $C_{u}$ are called canards. Canards may end at any place along the unstable portion of the slow manifold, turning abruptly and jumping parallel to the fast direction to one of the stable sheets of the slow manifold. If the trajectories return to the sheet of the slow manifold from which the canard started, we call them jump back canards. If they jump to the opposite sheet of the slow manifold from their origin, we call them jump away canards. We call the trajectories that follow the unstable portion of the slow manifold to the fold line without jumping maximal canards. As $\varepsilon \rightarrow 0$, the initial points for a whole family of canards approach the same point. To represent this behavior in the reduced system, we allow the flow to have multiple trajectories emanating from the same point. Specifically, along a canard orbit, there are three possibilities at each time: continuing to flow along the canard or jumping to either side of the unstable sheet of the slow manifold. We describe in section 3 how the dynamics of the canard orbits can be incorporated into extensions of the half return map. Formally, we extend the concept of a discrete dynamical system from maps of a space to 
relations for that space. Iterations of one dimensional mappings are sufficiently constrained in their properties that we can still analyze the properties of the dynamical systems defined by these relations. Intuitively, we regard the extensions as segments of the graph of $H$ whose domain has infinitesimal width and large slope.

The extended half return map is essentially a one dimensional discrete dynamical system. The dynamics of one dimensional mappings can be analyzed in terms of symbolic dynamics (or kneading theory) [20]. Briefly, the domain of a mapping is partitioned into intervals of monotonicity. These are intervals on which the map is continuous and strictly increasing or decreasing. A symbol is assigned to each interval, and trajectories are mapped to symbol sequences that encode which intervals in the partition contain successive iterates of the initial point. The extreme values of the intervals of monotonicity are called critical values and their symbol sequences are called the kneading data of the mapping. The full set of symbol sequences associated with trajectories of the mapping can be reconstructed from the kneading data by simple rules. This allows one to almost fully characterize the nonwandering set of mappings from the kneading data, a task that can be completed within certain classes of mappings [12]. Bifurcations in a family of mappings occur when the kneading data change. This happens in a fashion that is constrained by the one dimensionality of the dynamics, and a great deal is known about "universal" patterns that occur in the bifurcations of one dimensional maps [8]. Each extreme value can be regarded as an independent parameter in determining the dynamics of maps with specified critical points and discontinuities. We apply this theory to the extended half return map of the reduced mapping for the forced van der Pol oscillator. Given the number of discontinuities of $H$, there are fewer types of topological equivalence classes for the extended half return maps than one might expect from the kneading theory. The half return maps may have several points of discontinuity, but there are only two possible values for left and right limits of $H$ as $\theta$ approaches a point of discontinuity. Moreover, the endpoints of the canard extensions of the intervals are mapped to the same point by $H$. Additionally, there is at most one interior local maximum and one interior local minimum of $H$, so these are the only extreme points that are not points of discontinuity. These points exist precisely when $|a|>2$ and the points of tangency of the slow flow with $S_{ \pm 2}$ do not lie in the stable manifold of the folded saddle.

The existence of the chaotic invariant sets in the forced van der Pol system was discovered by Cartwright and Littlewood $[6,7,18,19]$ - a seminal event in the history of dynamical systems. One of the objectives of this work is to develop a more comprehensive understanding of the nonwandering sets of the forced van der Pol system and how these change with the parameters $(a, \omega, \varepsilon)$. Vestiges of the chaotic invariant sets remain in the dynamics of the extended half return maps. Based upon analysis of $H$ and its extensions, we give a conjectural description of the complete nonwandering sets of the forced van der Pol oscillator for many parameter values where there are chaotic invariant sets. When the trajectories of the critical values of $H$ approach stable periodic orbits and there are no neutrally stable periodic orbits, we believe that the dynamics of the van der Pol system for nearby parameter values are structurally stable. We identify simple examples where the analysis of the half return map indicates that this behavior occurs and describe the topological equivalence classes of the nonwandering sets of these examples as subshifts of finite type. We present a conjectural picture of the relationship between the nonwandering sets of the extended $H$ and the nonwandering sets of 
the system (1.1). Additional analysis is needed to prove these conjectures, but our description gives a detailed geometric perspective on the classical studies of Cartwright and Littlewood that is global in both the phase space and parameter space of the forced van der Pol oscillator.

2. Canards in the reduced system. The reduced system defined in [14] is a hybrid dynamical system given by the slow flow of system (1.2) in the regions of the $(\theta, x)$ cylinder with $|x| \geq 1$ together with discrete transition maps from the circles $S_{ \pm 1}$ to the circles $S_{\mp 2}$, since $y=x^{3} / 3-\left.x\right|_{x= \pm 1}=\mp 2 / 3=x^{3} / 3-\left.x\right|_{x=\mp 2}$. When $a>1$, there are equilibrium points of the slow flow at the points $(\theta, x)=\left( \pm \sin ^{-1}(1 / a) / 2 \pi, \pm 1\right)$ on the boundary of the region in which the reduced system is defined. Two of these equilibrium points are saddles for the slow flow. The other two equilibrium points are stable nodes if $1<a<\sqrt{1+1 /(16 \pi \omega)^{2}}$ and stable foci if $a>\sqrt{1+1 /(16 \pi \omega)^{2}}$. These equilibria are called folded equilibria because they lie on the fold curves of the critical manifold and do not correspond to equilibria of the van der Pol system (1.1).

We examine the folded saddle $p_{1 s}=\left(\theta_{1 s}, 1\right)$ lying on $x=1$ and call its stable and unstable manifolds for the slow flow (1.2) $W_{s}$ and $W_{u}$, respectively. We denote the first intersection of $W_{u} \cap C_{s}$ by $p_{1 u}=\left(\theta_{1 u}, 1\right)$ and the first intersection of $W_{s} \cap C_{u}$ with one of the circles $S_{ \pm 1}$ by $p_{m}=\left(\theta_{1 m}, \pm 1\right)$. In the van der Pol system (1.1), some trajectories arriving in the vicinity of $p_{1 s}$ continue a slow motion near $W_{s} \cap C_{u}$ rather than jumping to the stable sheet of the critical manifold near $S_{-2}$. These trajectories are called canards. Benoît [4] analyzed the dynamics of folded saddles in slow-fast systems with two slow variables and one fast variable. His analysis describes the limit properties of canard trajectories as $\varepsilon \rightarrow 0$. (See also the discussions of Mischenko et al. [21] and Szmolyan and Wechselberger [23].) We summarize here the results that provide the foundation for our analysis of canards in the reduced system.

Fix a neighborhood of $p_{1 s}$ that contains a ball $B$ of radius $O(1)$ centered at the folded saddle. Benoit proves that there is a constant $c>0$ so that the sets of trajectories that remain within distance $O(\varepsilon)$ from $C \cap B$ form two bundles, each with a cross-section at the folded saddle whose diameter is $O(\exp (-c / \varepsilon))$. As $\varepsilon \rightarrow 0$, one of these bundles of slow orbits approaches $W_{s}$ and the other approaches $W_{u}$. Note that the time rescaling used to obtain the slow flow reverses time on $C_{u}$, so the trajectory in $W_{s} \cap C_{u}$ lies close to trajectories of the van der Pol system flowing away from the folded saddle and not toward it. Benoît further proves that the strong stable manifold of $W_{s} \cap C_{u}$ and the strong unstable manifold of $W_{s} \cap C_{u}$ meet transversally near the folded saddle. Denote by $\gamma_{u}$ the segment of $W_{s} \cap C_{u}$ between $p_{1 s}$ and $p_{1 m}$. Given a point $p \in \gamma_{u}$, Benoit's results imply that there are canards in the van der Pol system that emanate from the folded saddle and then jump from $\gamma_{u}$ in an $O(\varepsilon)$ neighborhood of $C_{u}$ near $p$. Thus we model the limits of canards in the reduced system as the canard umbra of $\gamma_{u}$. We define the umbra of $\gamma_{u}$ to be a pair of curves on $C_{s}$ swept out by the intersection of lines parallel to the $x$ axis through points of $\gamma_{u}$. If $p=\left(x_{0}, y, \theta\right)$, the corresponding points on the umbra have the form $\left(x_{1}, y, \theta\right)$, where $x_{1}^{3}-3 x_{1}=x_{0}^{3}-3 x_{0}$ or $x_{1}^{2}+x_{0} x_{1}+x_{0}^{2}-3=0$.

Figure 1 shows structures of the reduced system for parameter values $(a, \omega)=(1.1,1.57)$. Since trajectories make more than a single circuit in the $\theta$ direction, two fundamental domains in $\theta$ are plotted, with repetitions of the folded equilibria drawn. The stable and unstable manifolds of the folded saddle are drawn in black. The segment $\gamma_{u}$ of $W_{s}$ is drawn as a heavy black line on one copy of $W_{u}$. The two umbra of $\gamma_{u}$ are drawn as heavy magenta and 


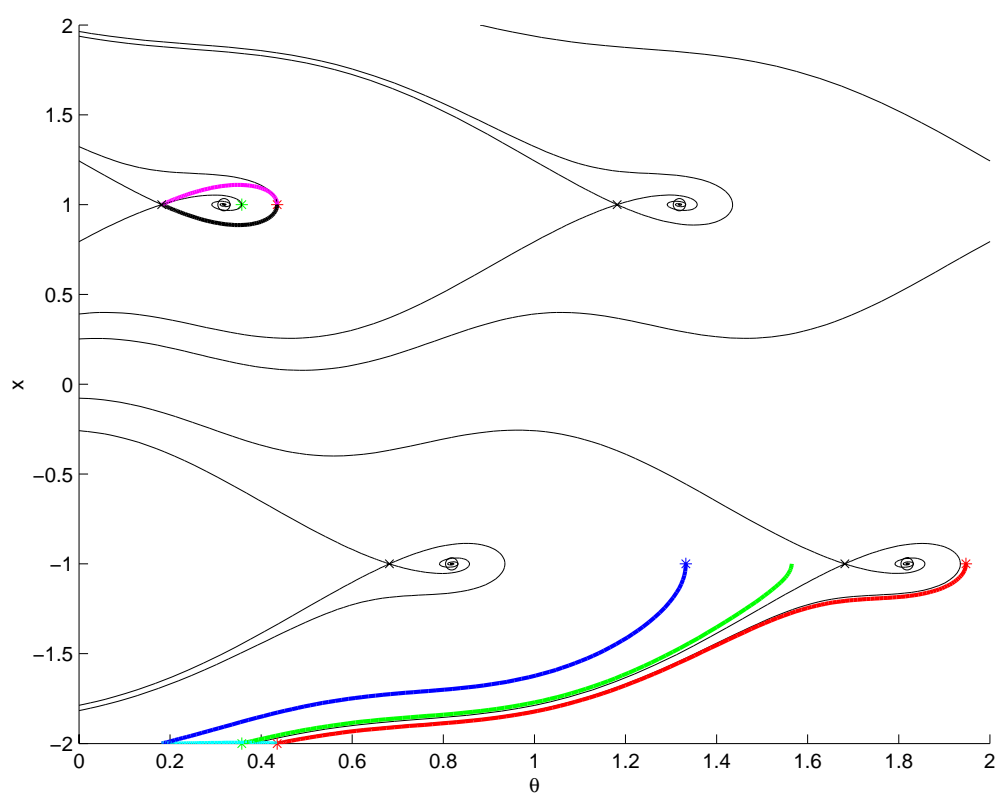

Figure 1. The phase portrait of the reduced system for the parameter values $(a, \omega)=(1.1,1.57)$. The stable and unstable manifolds of the saddle are drawn in black. The segment $\gamma_{u}$ is represented by the thick black line and the two umbral curves are drawn in magenta and cyan. The blue and red lines represent the trajectories at the ends of the cyan umbral curve and the green line is the trajectory of $p_{1 u}$.

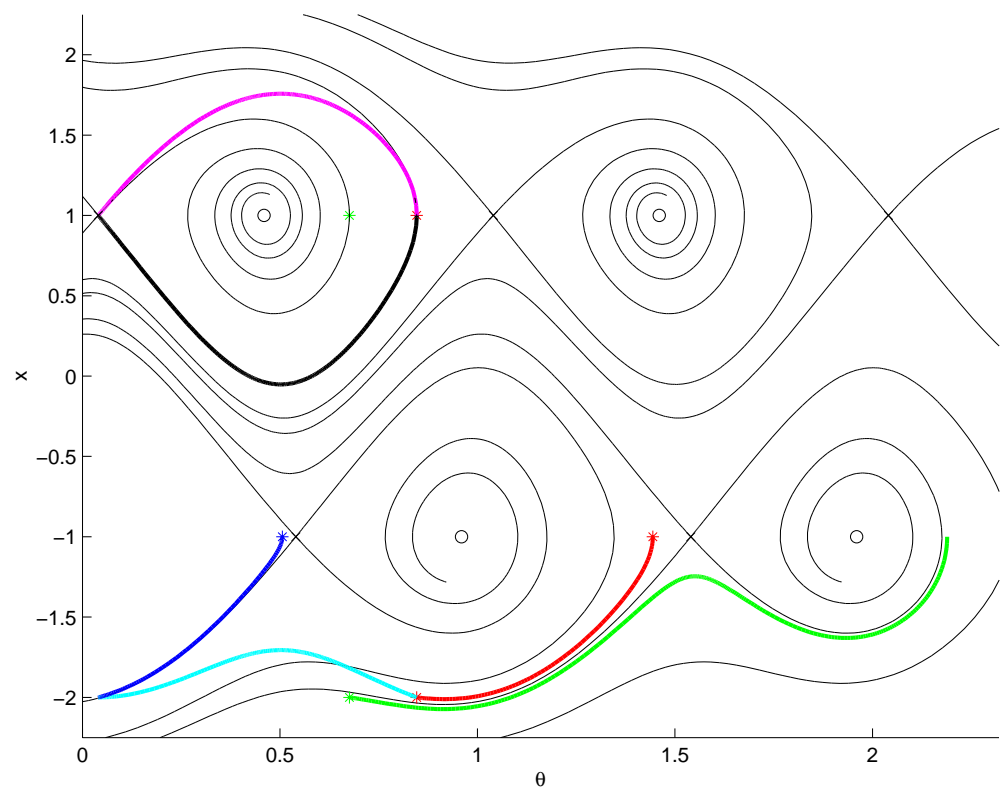

Figure 2. The phase portrait of the reduced system for the parameter values $(a, \omega)=(4,1.51)$. Note that the color coding is the same as represented in Figure 1. 


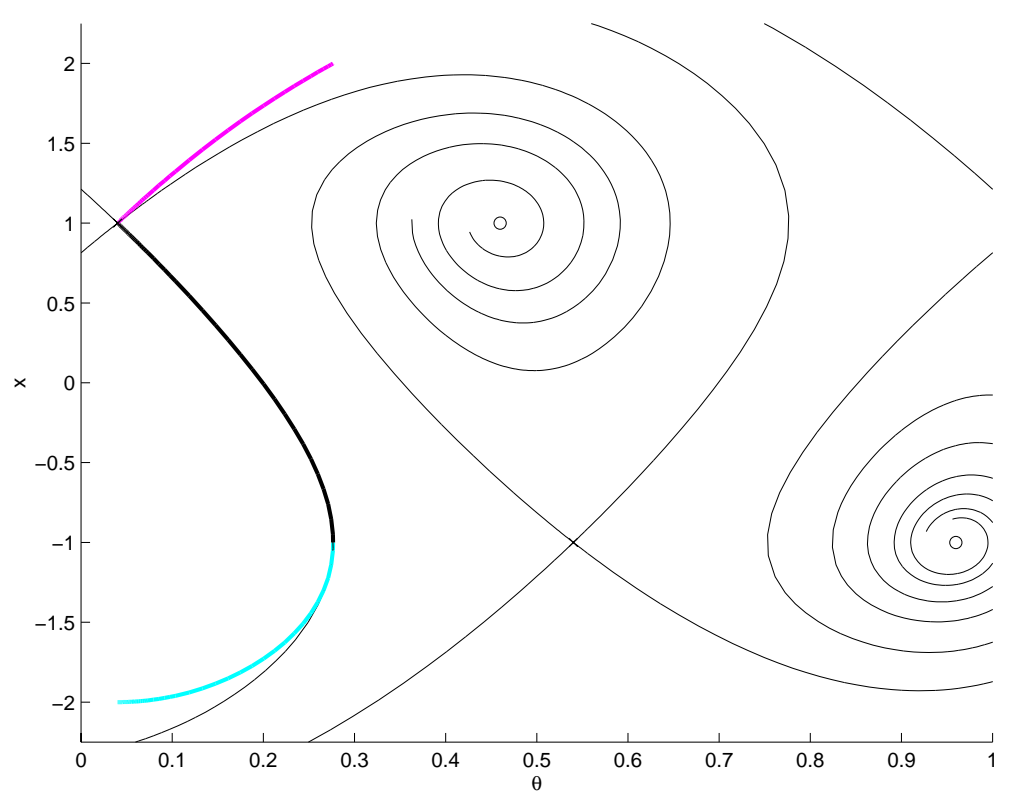

Figure 3. The phase portrait of the reduced system for the parameter values $(a, \omega)=(4,0.5)$. Note that the color coding is the same as represented in Figure 1.

cyan curves. The point $p_{1 u}$ at the intersection of $W_{u}$ with $S_{1}$ is a green asterisk, and the continuation of $p_{1 u}$ in the reduced system is plotted in green to its intersection with $S_{-1}$. The trajectories starting at the ends of the umbra in the region $x \leq-1$ are drawn in blue and red. Figure 2 shows structures of the reduced system for parameter values $(a, \omega)=(4,1.51)$. The color coding of the structures is the same as in Figure 1. Finally, Figure 3 shows structures of the reduced system for parameter values $(a, \omega)=(4,0.5)$. Note that the maximal canard point for these parameter values is on $S_{-1}$ rather than on $S_{1}$ as it is in Figures 1 and 2.

3. The extended half return map. This section defines a multivalued extension to the half return map $H$ whose new values reflect the limit properties of canards in the van der Pol system (1.1). We assume that we are in the parameter region in which the maximal canard point $p_{m}$ is on $S_{1}$ rather than on $S_{-1}$. The definition of the extension is not completely straightforward because the canards with jumps that decrease the value of $x$ yield trajectories that hit the cross-section $S_{-1}$ before they hit $S_{1}$. Therefore, we introduce an artifice of flowing backward along the slow flow from the canard umbra in $x<-1$ to the cross-section $S_{-2}$, where we can apply the symmetry to obtain a value for the extended half return map. We state and prove a fundamental lemma before describing details of this construction.

Lemma 3.1. The slow flow vector field is transverse to the umbra of the canard curve $\gamma_{u} \subset W_{s} \cap C_{u}$.

Proof. Let $\left(\theta, x_{0}\right)$ be a point on $\gamma_{u} \subset W_{s} \cap C_{u}$. Then the corresponding points on the umbra have the form $\left(\theta, x_{1}\right)$ with $x_{1}^{2}+x_{0} x_{1}+x_{0}^{2}-3=0$. The slope of the vector field at $\left(\theta, x_{1}\right)$ is

$$
\sigma_{1}=\frac{-x_{1}+a \sin (2 \pi \theta)}{\omega\left(x_{1}^{2}-1\right)},
$$


while the slope of the umbra is

$$
\tau_{1}=\left(\frac{d x_{1}}{d x_{0}}\right)\left(\frac{-x_{0}+a \sin (2 \pi \theta)}{\omega\left(x_{0}^{2}-1\right)}\right)=\left(\frac{x_{1}+2 x_{0}}{2 x_{1}+x_{0}}\right)\left(\frac{-x_{0}+a \sin (2 \pi \theta)}{\omega\left(x_{0}^{2}-1\right)}\right) .
$$

Simplifying $\sigma_{1}-\tau_{1}$ (with the help of the computer program Maple), we find that

$$
\sigma_{1}-\tau_{1}=\frac{ \pm 3}{\omega \sqrt{12-3 x_{0}^{2}}} \neq 0
$$

the sign depends upon which root $x_{1}$ we take. Since the two slopes are different, the vector field at $\left(\theta, x_{1}\right)$ is transverse to the umbral curves.

This lemma implies that distinct points on each umbral curve lie on distinct trajectories. To define the extension of the half return map $H$ for the umbral curve that lies in the half cylinder $x \geq 1$, we follow the trajectories of the slow flow from a point $\left(\theta_{0}, x_{0}\right)$ on the umbral curve to its intersection with $S_{1}$ at the point $\left(\theta_{1}, 1\right)$ and then define $H$ for this canard trajectory to be $\theta_{1}+0.5$. This gives the same value of $\theta$ as jumping from $\left(\theta_{1}, 1\right)$ to $\left(\theta_{1},-2\right)$ and then applying the symmetry of the slow flow. The extension of $H$ adds a vertical segment to its graph, making $H$ multivalued. The transversality lemma above implies that each value $\theta_{1}+0.5$ on the extended graph of $H$ corresponds to orbits containing a canard with a specific jump point, namely, $\left(\theta_{0}, x_{0}\right)$. Thus we shall regard the extension of $H$ as a monotone segment of infinite slope when analyzing the symbolic dynamics of the extended $H$ in the next section. With this definition, we obtain a limit of trajectories in the van der Pol system consisting of a canard that flows to a jump point along $W_{s}$, followed by a jump to $\left(x_{0}, x_{0}^{3} / 3-x_{0}, \theta_{0}\right)$ on the critical manifold, followed by a trajectory segment of the slow flow that reaches $x=1$ at $\left(1,-2 / 3, \theta_{1}\right)$ and then jumps to $x=-2$.

Defining the extension of $H$ for the umbral curve in the half cylinder $x \leq-1$ is less straightforward. When points jump from $W_{s}$ to a point $\left(\theta_{0}, x_{0}\right)$ on the umbral curve in the half cylinder $x \leq-1$, the trajectory from $\left(\theta_{0}, x_{0}\right)$ has "skipped" an intersection with the circle $S_{-2}$. The definition of the half return map was based upon trajectories reaching the half cylinder $x \leq-1$ at $x=-2$ so that the symmetry could be used to map them back to $S_{2}$. We resolve this difficulty by following the trajectory from $\left(\theta_{0}, x_{0}\right)$ backward to its first intersection with the circle $S_{-2}$ at $\left(\theta_{1},-2\right)$. The extension of $H$ is defined to give a value $\theta_{1}+0.5$ for this point on the umbral curve. The qualitative properties of the backward flow from the umbral curve to $S_{-2}$ depend upon whether there are zero, one, or two tangencies of the slow flow with $S_{-2}$ in the interval $\left(\theta_{1 s}, \theta_{m}\right)$ "covered" by the umbral curve. The tangencies occur at values of $\theta=\arcsin (-2 / a)$. If there are no tangencies, then the set of values for $\theta_{1}+0.5$ is the entire interval $\left(\theta_{1 s}, \theta_{m}\right)$. If there is a single point of tangency, then the slow flow has $x^{\prime}\left(\theta_{m},-2\right)<0$, and the set of values for $\theta_{1}+0.5$ is a connected interval whose right endpoint is the first intersection of the backward trajectory of the slow flow through $\left(\theta_{m},-2\right)$ with $S_{-2}$. If there are two points of tangency, then the set of $\theta_{1}+0.5$ consists of two intervals with a gap. The gap is an interval whose endpoints both lie on the slow flow trajectory passing through the right-hand tangency point on $S_{-2}$. In this case, note that the $H$ image of this pair of intervals is connected; the endpoints of the gap both have the same $H$ value. The transversality lemma again implies that each value $\theta_{1}+0.5$ on the extended graph of $H$ 


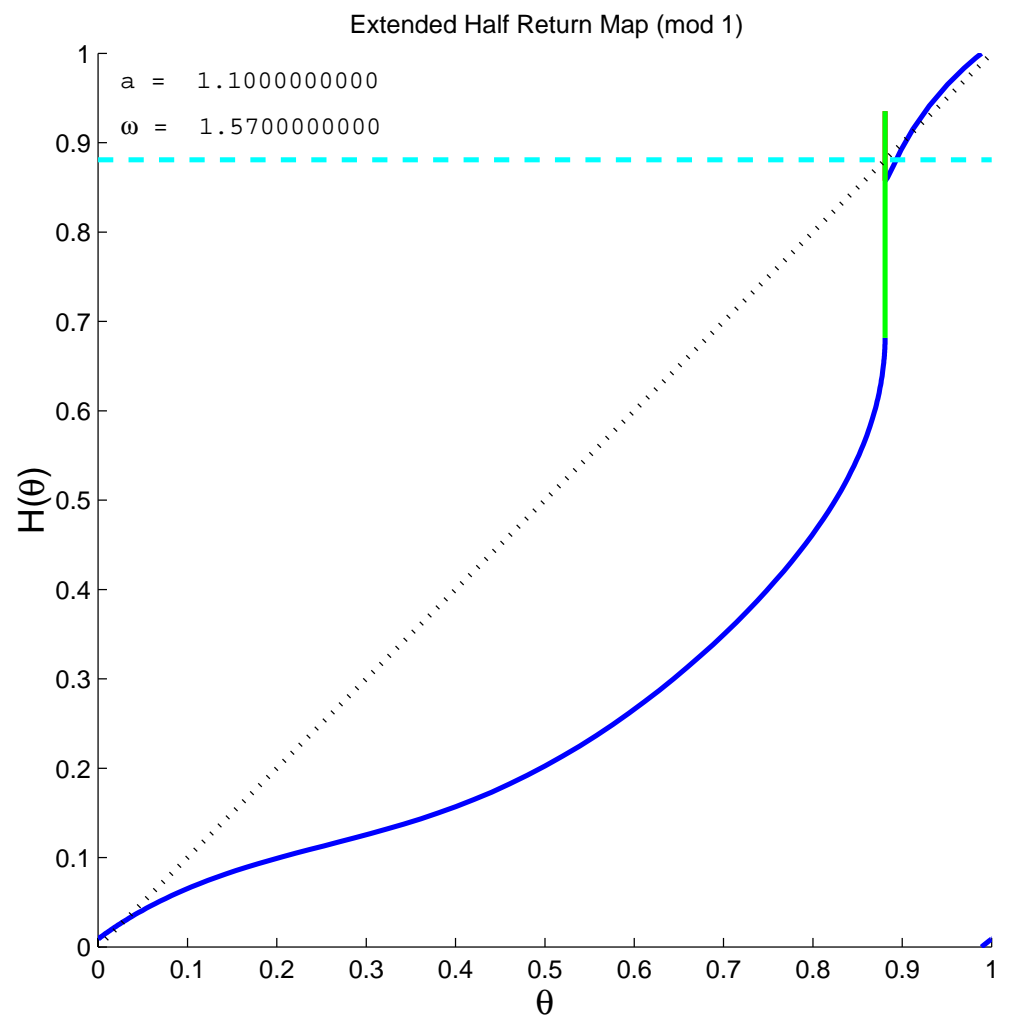

Figure 4. A plot of the extended half return map for the parameter values $(a, \omega)=(1.1,1.57)$. The canard extensions of $H$ are drawn as vertical segments.

corresponds to orbits containing a canard with a specific jump point. The limit trajectory for the van der Pol system consists of a canard that flows to a jump point along $W_{s}$, followed by a jump to $\left(x_{0}, x_{0}^{3} / 3-x_{0}, \theta_{0}\right)$ on the critical manifold, followed by a trajectory segment that lies on the trajectory of the slow flow through $\left(-1,2 / 3, \theta_{1}\right)$, but with the initial point of the segment being $\left(x_{0}, x_{0}^{3} / 3-x_{0}, \theta_{0}\right)$ and the final point of the segment being on $S_{-1}$.

Figures 4, 5, and 6 show three examples of the extended half return map for parameter values $(a, \omega)=(1.1,1.57),(4,1.51)$, and $(1.02,1.19)$, respectively. When $1<a<2$, there is a single point of discontinuity and no local maximum or minimum of $H$. Therefore, the values of the maximal canards for the left and right extensions at the discontinuity point have equal values. The canard extensions of $H$ are drawn as (overlapping) red and green vertical segments. When $(a, \omega)=(1.1,1.57), H$ itself has two fixed points and there are two additional fixed points on the canard extensions.

At parameter values $(a, \omega)=(4,1.51), H$ has three points of discontinuity. It also has a local maximum and local minimum that are not at points of discontinuity. In the flow of the reduced system, $x^{\prime}<0$ at some of the points on the umbra of the canards in $x<-1$. Therefore, the values of the maximal canards on the extensions of $H$ do not agree. However, note that the images of the maximal canard values are the same - a point that lies on the branch of $H$ with a local minimum. Also, the canard extensions at each of the three discontinuity points 


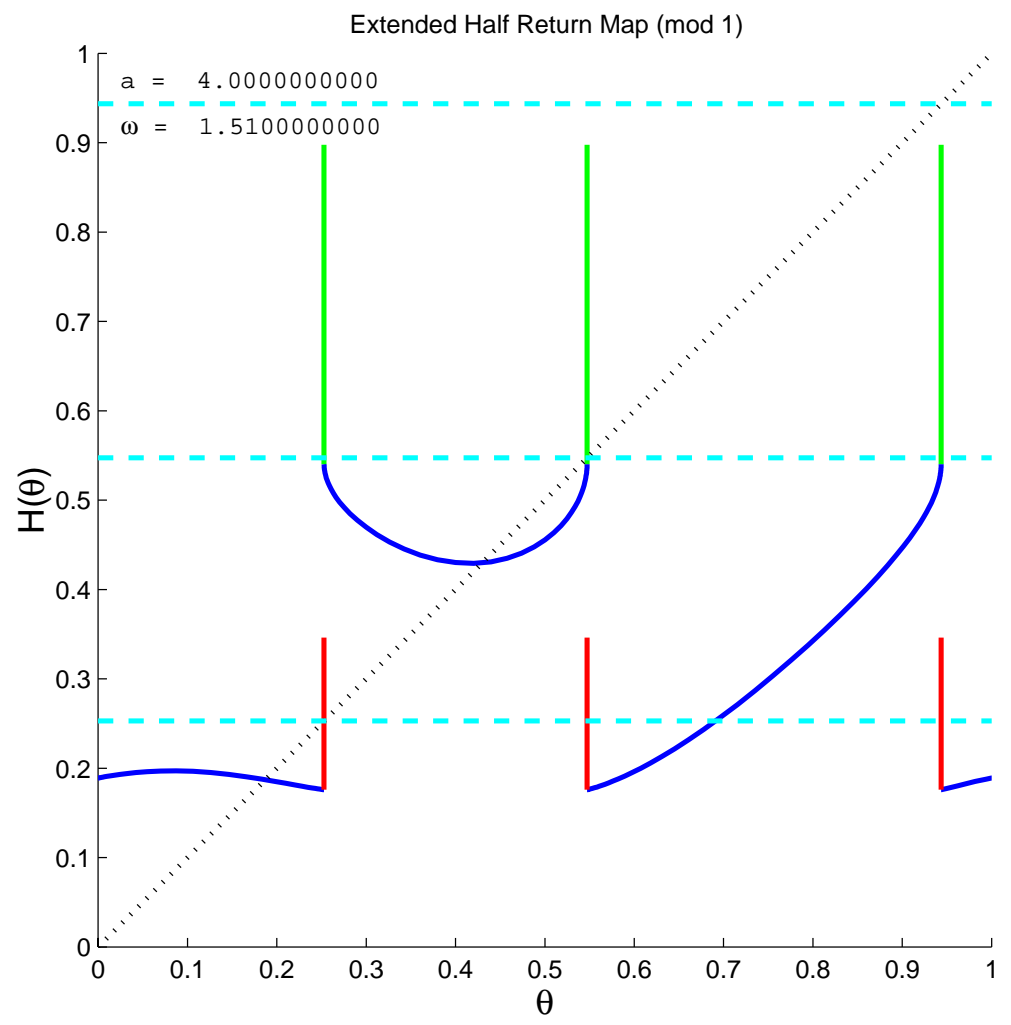

Figure 5. A plot of the extended half return map for the parameter values $(a, \omega)=(4,1.51)$. The canard extensions of $H$ are drawn as red and green vertical segments.

have the same length. Both the branch with a local minimum and the branch with a local maximum have stable fixed points. In addition there are two fixed points on two of the canard extensions.

At parameter values $(a, \omega)=(1.02,1.19), H$ has no fixed points at all. However, the second iterate of $H$, displayed in Figure 7 , has features that resemble the half return map for $(a, \omega)=(1.1,1.57)$. There are four fixed points of $H^{2}$ outside its canards, coming from two periodic orbits of period 2. In addition, $H^{2}$ has fixed points on each of its canard segments.

4. Poincaré maps. An alternative method for studying canard solutions of the forced van der Pol equation is to use the idea of Poincaré maps. We define a half Poincaré map $\Phi_{\varepsilon}$ for (1.1) and a corresponding singular half Poincaré map $\Phi_{0}$ for the reduced system. Let $\Sigma_{1}$ be the half cylinder $\{(x, y, \theta) \mid x=-1, y<2 / 3-\delta\}$ for some small $\delta>0$ (see Figure 8 ). The system (1.1) is invariant under the symmetry transformation $T(x, y, \theta)=(-x,-y, \theta+1 / 2)$. Let $\Sigma_{2}=T\left(\Sigma_{1}\right)$; that is, $\Sigma_{2}=\{(x, y, \theta) \mid x=1, y>-2 / 3+\delta\}$. As long as we consider $\varepsilon$ sufficiently small and parameter values $(a, \omega)$ in the interior of the region $M_{1}$ (defined in section 6), the flow map from $\Sigma_{1}$ to $\Sigma_{2}$ composed with the symmetry transformation is a well-defined map $\Phi_{\varepsilon}$ from $\Sigma_{1}$ to itself. In particular, the trajectories with initial conditions on $\Sigma_{1}$ cross the cylinder $x=1$ at points of $\Sigma_{2}$; canards that jump away do so with $y>-2 / 3+\delta$.

The singular half Poincaré map $\Phi_{0}: \Sigma_{1} \rightarrow \Sigma_{1}$ of the reduced system is composed of a 


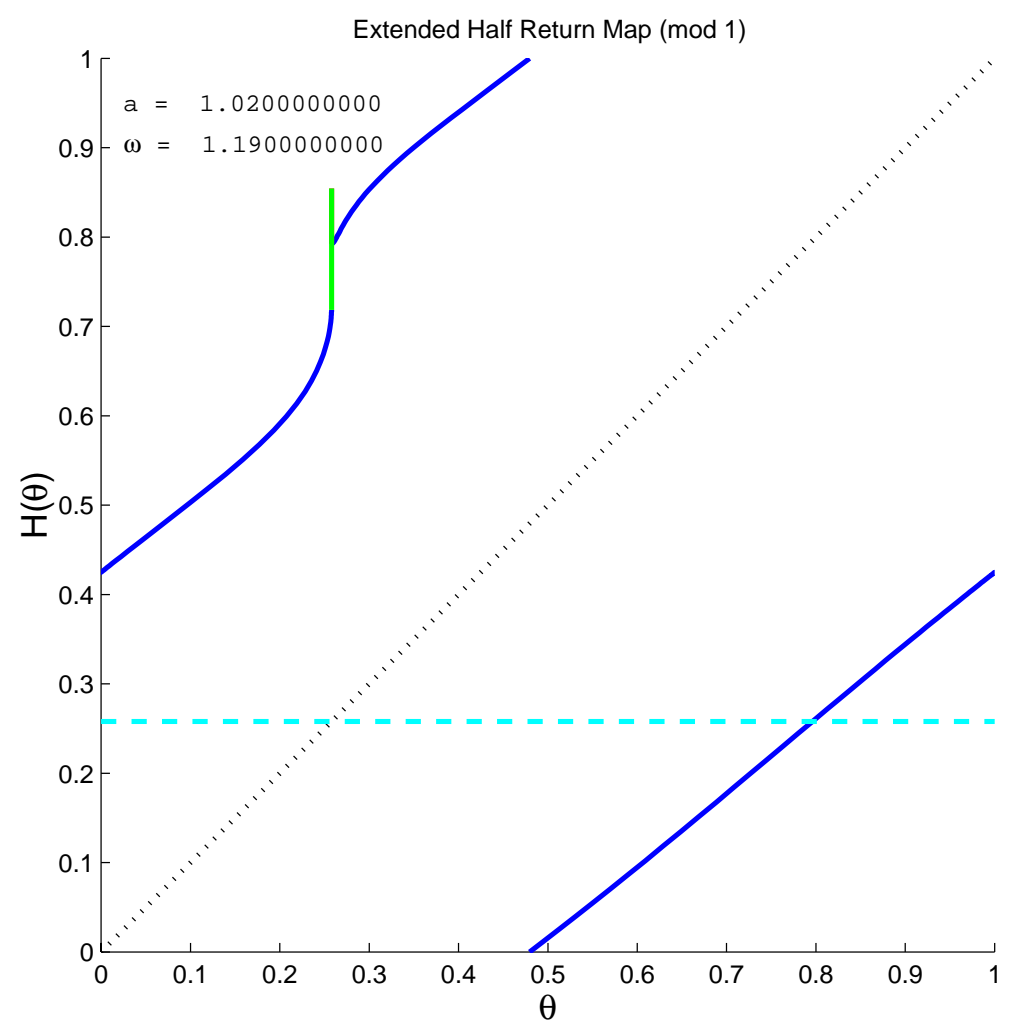

Figure 6. A plot of the extended half return map for the parameter values $(a, \omega)=(1.02,1.19)$. The canard extensions of $H$ are drawn as vertical segments.

projection along the $x$ direction onto $C_{s}$, followed by the slow flow on $C_{s}$ to the fold line $S_{-1}$, and a projection along the $x$ direction from $S_{-1}$ to $\Sigma_{2}$. The symmetry transformation $T$ maps $\Sigma_{2}$ back to $\Sigma_{1}$. This map is singular; all points in $\Sigma_{1}$ map to the line $x=-1, y=-2 / 3$ in $\Sigma_{1}$. This definition of $\Phi_{0}$ breaks down on the stable manifold of the folded saddle. These points do not return, but they have canard extensions that do. So we extend the definition of $\Phi_{0}$ to account for the canards. As explained above, canards form on trajectories sufficiently close to the stable manifold of the saddle. In the van der Pol flow (1.1), let $\kappa_{\varepsilon} \subset \Sigma_{1}$ be the set of points for which canards will form during the flow from $\Sigma_{1}$ to $\Sigma_{2}$. When these trajectories finally cross $\Sigma_{2}$, they have either jumped from a point near $C_{u}$ to $\Sigma_{2}$ without returning to a neighborhood of $C_{s}$ (the jump away canards), or they have jumped back to a neighborhood of $C_{s}$ and then jumped a second time, passing the fold $S_{-1}$ between $\theta_{1 u}$ and $\theta_{m}$ (the jump back canards). For $0<\varepsilon \ll 1, \kappa_{\varepsilon}$ is a thin strip. For the reduced system and $\varepsilon=0$, we define $\kappa_{0}$ to be curve in $\Sigma_{1}$ that projects onto the stable manifold of the saddle. To account for canards in $\Phi_{0}$, we make $\Phi_{0}$ a multivalued relation. For any $p \in \kappa_{0}$, we define $\Phi_{0}(p)$ to be the curve made up of the projections of $\gamma_{u}$ onto $\Sigma_{2}$ (representing jump away canards) and the projection of the segment $\left(\theta_{1 u}, \theta_{m}\right)$ on the fold line $S_{-1}$ onto $\Sigma_{2}$ (representing jump back canards). In Figure 9, we give a qualitative sketch of such a Poincaré map. Figures 10 and 11 show the map for $(a, \omega)=(1.1,1.57)$ and $(a, \omega)=(4.0,1.51)$. 


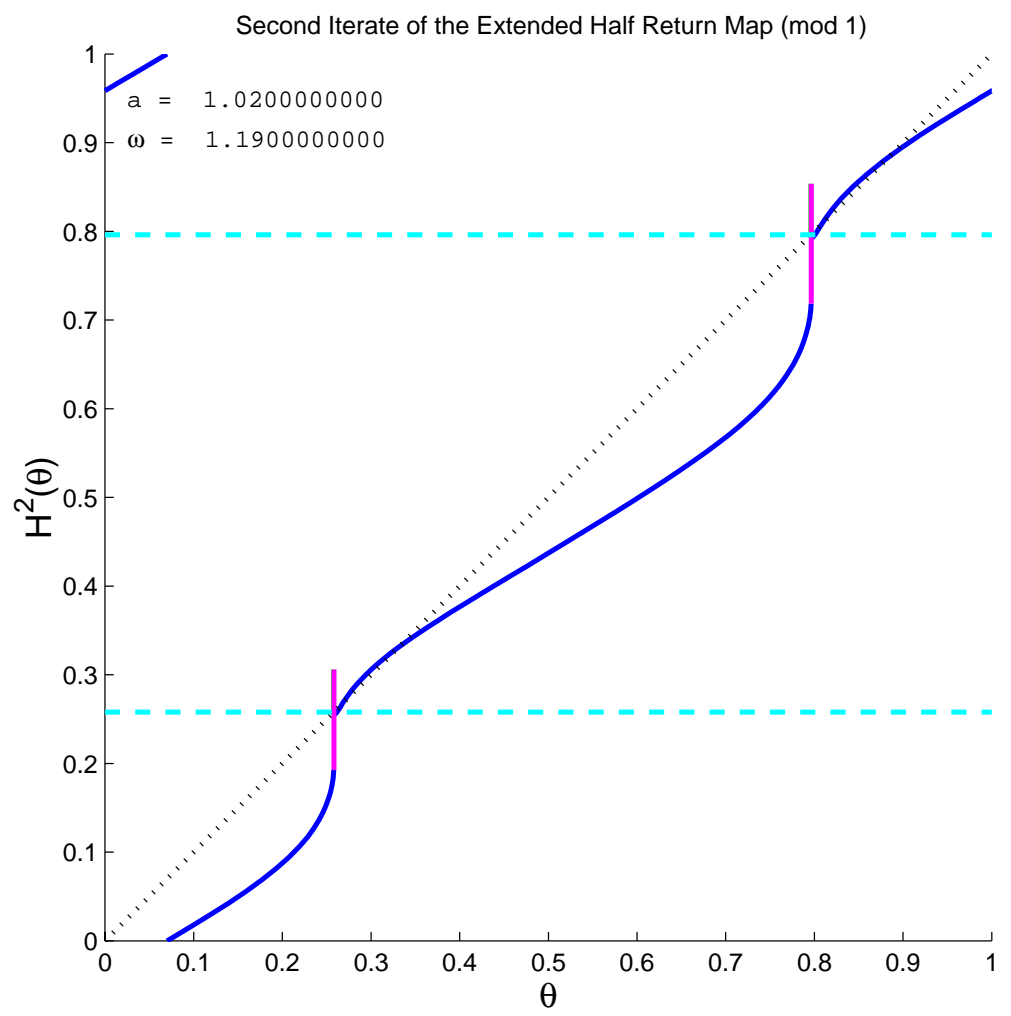

Figure 7. A plot of the second iterate of the extended half return map for the parameter values $(a, \omega)=$ $(1.02,1.19)$.

In Figure 12, we show four examples of singular Poincaré maps where there are two canard fixed points. These come from parameter values $(a, \omega)$ where we expect the van der Pol system will have chaotic invariant sets for small $\varepsilon>0$. The stable manifolds (red) and images of the canards (cyan) give approximations to the expected directions of the stable and unstable bundles in a hyperbolic structure for these invariant sets. In the next section, we use the insight obtained from the singular half Poincaré map in numerical calculations with the three dimensional system with small values of $\varepsilon>0$.

5. Symbolic dynamics. The extensions to the half return map defined in the previous section consist of two vertical segments that we add to the graph of $H$ at each point of discontinuity. Here we explain how to use symbolic dynamics of one dimensional maps to analyze the extended half return map. The graph of the map is a multivalued function, or relation, so we define trajectories for such objects. We identify sets of trajectories that can be used to reconstruct trajectories with canards in the reduced system that we conjecture are limits as $\varepsilon \rightarrow 0$ of trajectories with canards in the forced van der Pol system (1.1). We begin by recalling some of the topological theory of iterations of one dimensional maps, following Milnor and Thurston [20]. We then describe how we extend this theory to account for multivalued maps that have vertical "monotone" segments and discontinuities like those of our extended half return map. We end the section with descriptions of the nonwandering 


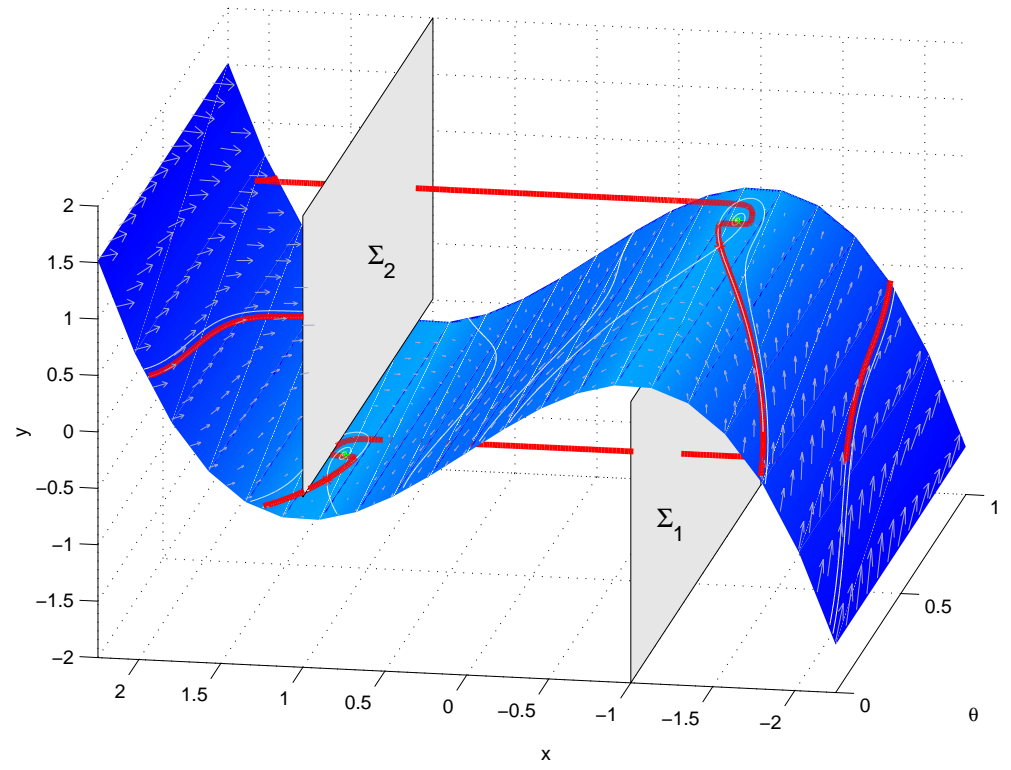

Figure 8. The blue surface is the critical manifold $C$. The white lines and grey arrows in $C$ are integral curves and the vector field of the slow flow, respectively. The red curve is an unstable periodic orbit of (1.1), computed by AUTO, for the parameter values $\varepsilon=0.0001, a=1.0888485842$, and $\omega=1.55$.

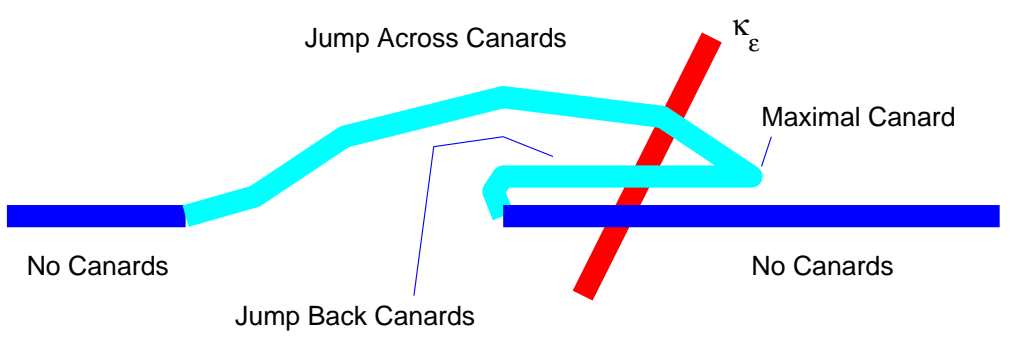

Figure 9. A cartoon of the image of $\Sigma_{1}$ under the Poincaré map $\Phi_{\varepsilon}$. The union of the dark blue and cyan curves represents $\Phi_{\varepsilon}\left(\Sigma_{1}\right)$. The red line represents $\kappa_{\varepsilon}$, the subset of $\Sigma_{1}$ for which canards form during the flow from $\Sigma_{1}$ to $\Sigma_{2}$, and the cyan segment represents $\Phi_{\varepsilon}\left(\kappa_{\varepsilon}\right)$. (The vertical separation between the cyan segment labeled "Jump Back Canards" and the dark blue segment on the right is greatly exaggerated.)

sets of the examples displayed in the previous section.

Let $H: I \rightarrow I$ be a piecewise monotone map of the interval $I$. We also allow the possibility that $I$ is the circle $S_{1}$. Here piecewise monotone means that there are points $c_{0}, \ldots, c_{n}$ such that, on each element of the partition $I=I_{1} \cup I_{2} \cup \cdots \cup I_{n} ; I_{j}=\left[c_{j-1}, c_{j}\right]$ (with $c_{0}=c_{n}$ in case $I$ is a circle), $H$ has a continuous strictly monotone extension to each $I_{j}$. Note that this definition allows $H$ to be discontinuous at the $c_{j}$. We assign symbol sequences, called itineraries, to each $x$ by $A(x)=\left\{a_{i}\right\}$ with $a_{i}=j$ when $H^{i}(x) \in I_{j}$. The shift map $\sigma$ on sequences is defined by $\sigma(\bar{a})=\bar{b}$ when $b_{i}=a_{i+1}$. The best way to treat the mesh points $c_{j}$ of the partition in this definition is problematic. Since we shall look at multivalued extensions of the map that send endpoints to entire intervals, this issue is discussed at length below. Each 


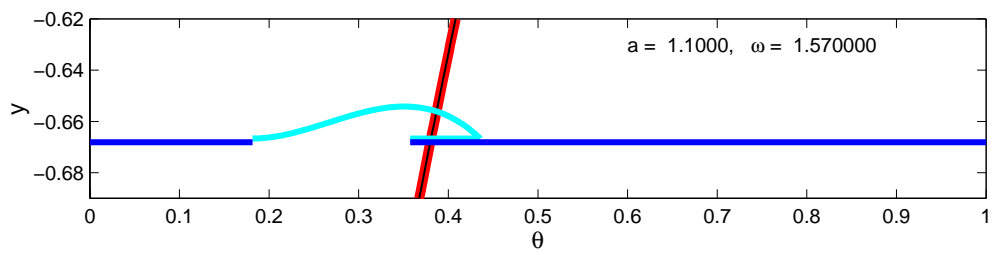

Figure 10. The singular Poincaré map for $(a, \omega)=(1.1,1.57)$. The red curve is $\kappa_{0}$.

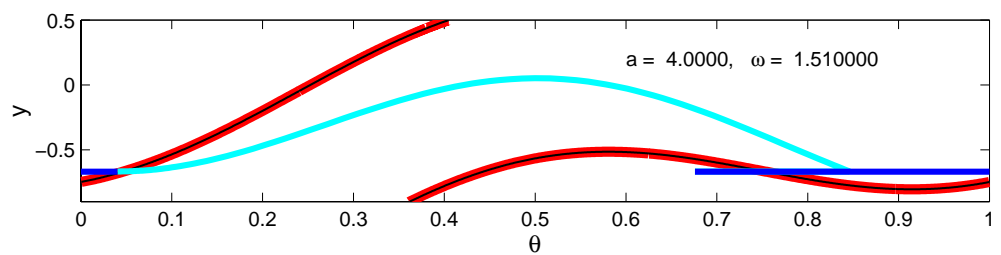

Figure 11. The singular Poincaré map for $(a, \omega)=(4.0,1.51)$. The red curve is $\kappa_{0}$.
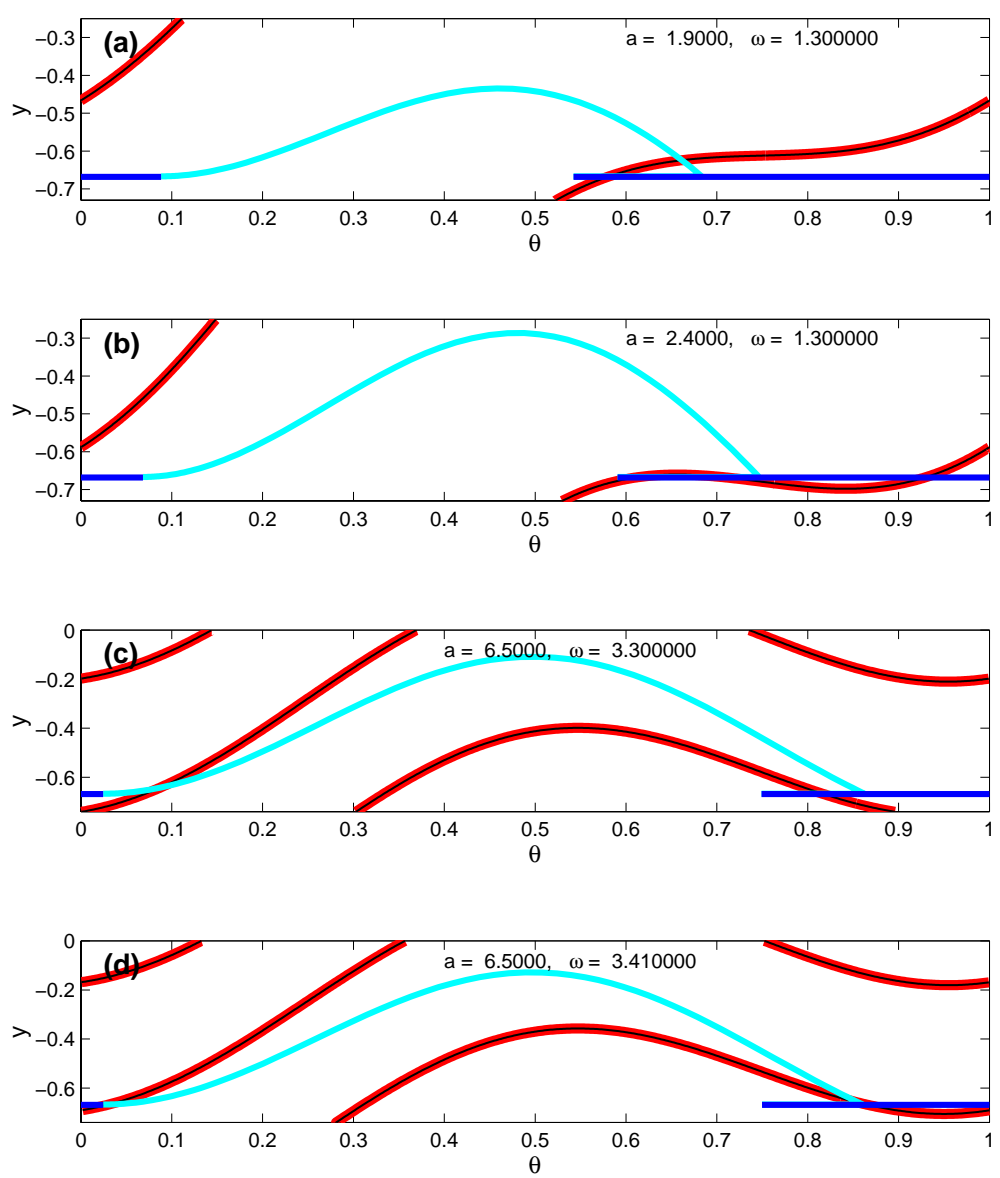

Figure 12. Singular Poincaré maps that show the four cases where $\Phi_{0}\left(\kappa_{0}\right)$ intersects $\kappa_{0}$ twice. 
interval $I_{j}$ has a sign $s(j)= \pm 1$ defined by whether the map $H$ is increasing or decreasing on $I_{j}$. These signs are used to define an order on itineraries: $\left\{a_{i}\right\}<\left\{b_{i}\right\}$ if the sequences differ and for the first index $k$ with $a_{k} \neq b_{k}, s\left(a_{0}\right) \cdots s\left(a_{k-1}\right) a_{k}<s\left(b_{0}\right) \cdots s\left(b_{k-1}\right) b_{k}$. Note that the two signs in this inequality are the same since the sequences agree up to their $k$ th terms. The map $H^{k}$ is increasing or decreasing on the interval

$$
\bigcap_{i=0}^{k-1} H^{-i}\left(I_{A_{i}}\right)
$$

as $s\left(a_{0}\right) \cdots s\left(a_{k-1}\right)= \pm 1$. This implies that the order of itineraries is consistent with the order of points of the interval; if $x<y$, then $A(x) \leq A(y)$.

There is a complementary theorem to the result that the ordered itineraries are consistent with the linear order of points on the interval. This theorem almost classifies the map $H$ up to topological equivalence. The points with the same itinerary are called monotone equivalence classes. These classes are connected sets: either points or intervals. The itineraries of the critical values $H\left(c_{i}\right)$ are called the kneading data for the map. The kneading data constrains the itineraries of other trajectories. If a point $H^{i}(x) \in I_{j}$, then $s(j) A\left(H\left(c_{j-1}\right)\right) \leq$ $s(j) A\left(H^{i+1}(x)\right) \leq s(j) A\left(H\left(c_{j}\right)\right)$. The following theorem states that all sequences of addresses that satisfy strong versions of these inequalities are itineraries.

Theorem 5.1 (see [20]). If a sequence of symbols $\bar{a}$ satisfies the inequalities $s(j) A\left(H\left(c_{j-1}\right)\right)$ $<s(j) \sigma^{i+1}(\bar{a})<s(j) A\left(H\left(c_{j}\right)\right)$ when $a_{i}=j$, then there is a point $x$ whose itinerary $i s \bar{a}$.

Thus the kneading data essentially characterizes the monotone equivalence classes of $H$. If we further know which monotone equivalence classes are intervals and which are points, this determines the topological equivalence class of the mapping. Thus, in classifying piecewise monotone one dimensional maps up to topological equivalence, the kneading data are the essential invariants.

We want to apply the kneading theory described above to the multivalued extended half return maps defined in the previous section. To accomplish this, we imagine that the extensions of the map are made by inserting intervals of infinitesimal length at the discontinuities of the map and think of the extensions as functions of positive or negative slope on these infinitesimal intervals. Formally, we generalize the definition of a discrete dynamical system to multivalued functions.

Given a set $X$, the graph of a function $f: X \rightarrow X$ is a subset $G \subset X \times X$ with the property that for each $x \in X$, there is exactly one element of $G$ whose first element is $x$. We relax this requirement and define a discrete dynamical system for multivalued functions, defined as relations $G \subset X \times X$ with the property that the projection of $G$ onto its first component is onto; i.e., every $x \in X$ is the first element of at least one pair $(x, y) \in G$. We define the trajectories of $G$ to be sequences $\left\{x_{i}\right\}$ such that $\left(x_{i}, x_{i+1}\right) \in G$ for all $i \geq 0$. If $G$ is the graph of a function $f: X \rightarrow X$, then each trajectory is the sequence of $f$ iterates of its first element. In our case, $X$ and $G$ must be chosen in a way that reflects that there are two overlapping vertical segments added to the graph of $H$ at each point of discontinuity. We do this by first setting $X$ to be the circle $S_{1}$, but with each critical point $p$ of $H$ "split" into left-hand and right-hand points $p_{l}$ and $p_{r}$. This makes $X$ a non-Hausdorff topological space. Next, the relation $G$ is defined by adding vertical segments $\left(p_{l}, q_{l}\right)$ and $\left(p_{r}, q_{r}\right)$ to the graph of $H$ at each discontinuity 
point $p_{l}, p_{r}$. If the discontinuity point of $H$ is not one of the two points $\arcsin (2 / a)$, then both of the branches of $H$ at the discontinuity point are increasing or decreasing. (If there is a point $(\arcsin (2 / a), 2)$ that lies in $W_{s}$, then $H$ has a continuous extension at this point.) Where the branches of $H$ are increasing, call $p_{r}$ the lower discontinuity point and $p_{l}$ the upper discontinuity point. Where the branches of $H$ are decreasing, call $p_{l}$ the lower discontinuity point and $p_{r}$ the upper discontinuity point. The limit value of $H$ at the lower discontinuity points is $\theta_{1 u}$, while the limit value of $H$ at the upper discontinuity points is $\theta_{1 s}$. At each lower discontinuity point $p_{*}$, we add the vertical segment $\left[\theta_{1 u}, \theta_{1 m}\right]$ to $G$. At each upper discontinuity point we add the vertical segment $\left[\theta_{1 s}, \theta_{2 u}\right]$, where $\theta_{2 u}$ depends on the sign of $\theta^{\prime}$ at $\left(-2, \theta_{m}\right)$. If $2+\arcsin \left(2 \pi \theta_{m}\right)>0$, we set $\theta_{2 u}=\theta_{1 m}$, while if $2+\arcsin \left(2 \pi \theta_{m}\right)<0, \theta_{2 u}$ is determined so that $\left(-2, \theta_{2 u}\right)$ is the first point on the backward trajectory of the slow flow (1.2) with initial point $\left(-2, \theta_{m}\right)$. This almost completes the definition of the relation $G$. The one remaining item in its definition is what happens at the inverse images of the critical points. If an inverse image of a critical point $p$ is a regular point $q$, we include both $\left(q, p_{l}\right)$ and $\left(q, p_{r}\right)$ in $G$. If an inverse image of a critical point $p$ is a critical point $q$, then we include only one of $\left(q, p_{l}\right)$ and $\left(q, p_{r}\right)$ in $G$. If $q$ is the left endpoint of an increasing branch or the right endpoint of a decreasing branch, then we include $\left(q, p_{l}\right)$ in $G$; otherwise, we include $\left(q, p_{r}\right)$ in $G$.

We next define a (cyclic) order on the relation $G$. We begin by extending the order of the circle to $X$ so that $p_{l}<p_{r}$ at each critical point. Next, we assign signs to the vertical segments in $G$ : upper discontinuity points of increasing branches and lower discontinuity points of decreasing branches are assigned positive slope with $\operatorname{sign} s=+1$, while the extensions of the graph of $H$ at upper discontinuity points of decreasing branches and lower discontinuity points of increasing branches are assigned negative slope with sign $s=-1$. Given points $\left(\theta_{1}, \psi_{1}\right)$ and $\left(\theta_{2}, \psi_{2}\right)$ in $G$, we say that $\left(\theta_{1}, \psi_{1}\right)<\left(\theta_{2}, \psi_{2}\right)$ if either $\theta_{1}<\theta_{2}$ or the points on the same vertical segment with sign slope $s$ and $s \psi_{1}<s \psi_{2}$.

We now define a symbolic dynamics for the relation $G$. Partition $G$ into segments $I_{1}, \ldots, I_{n}$ at critical points. At each critical point, points $\left(p_{l}, q\right)$ and $\left(p_{r}, q\right)$ belong to adjacent elements of the partition. Here a segment is defined relative to the order on $G$ : a segment includes all the pairs between any two pairs in the segment. The partition elements are closed curves in the plane which are "disjoint" if one distinguishes $p_{l}$ and $p_{r}$ at each critical point. As for a function, we define the itinerary $\bar{a}=A(\bar{x})$ for a trajectory $\bar{x}=\left\{x_{i}\right\}$ to be the symbol sequence with $a_{i}=j$ when $x_{i} \in I_{j}$. With these definitions, the itineraries of trajectories for the relation $G$ are determined precisely by inequalities on symbol sequences.

Theorem 5.2. A sequence of symbols $\bar{a}=\left\{a_{i}\right\}$ is the itinerary of a trajectory of $G$ if and only if it satisfies the inequalities $s(j) A\left(H\left(c_{j-1}\right)\right) \leq s(j) \sigma^{i+1}(\bar{a}) \leq s(j) A\left(H\left(c_{j}\right)\right)$ with $a_{i}=j$.

The proof remains the same as the proof of the original theorem. The key point is the observation that for any sequence satisfying the inequalities $s(j) A\left(H\left(c_{j-1}\right)\right) \leq s(j) \sigma^{i+1}(\bar{a}) \leq$ $s(j) A\left(H\left(c_{j}\right)\right)$, the sets

$$
\bigcap_{i=1}^{l} H^{-i}\left(I_{a_{i}}\right)
$$

form a decreasing sequence of nonempty compact connected subsets of $G$. Their intersection is a nonempty connected subset of the graph. 
If the critical points of a piecewise monotone map tend to stable periodic orbits and the nonwandering set has a hyperbolic structure [8], then the map is structurally stable. A map is structurally stable if perturbations of the map are all topologically equivalent; i.e., there are homeomorphisms that map trajectories of one map to trajectories of another. We comment here on the stability properties of $G$. If the trajectories of the critical points of $G$ tend to stable periodic orbits and the two points $(\arcsin (2 / a), 2)$ do not lie in $W_{s}$, then perturbations of the reduced system will have extensions with the same number of branches and the itineraries of the critical points will be unchanged. If, further, the "map" is expanding on the set of trajectories that do not tend to stable periodic orbits, then $G$ will be structurally stable: its nonwandering set will vary continuously with perturbation. The nonwandering set of a map with hyperbolic nonwandering set is topologically equivalent to a disjoint union of a finite number of stable periodic orbits and expanding subshifts of finite type. The nonwandering set of a structurally stable $G$ is similar, but with one difference that bears discussion. If there are distinct (but possibly coincident) critical points $p_{1}$ and $p_{2}$ so that the canard pairs $\left(p_{1}, p_{1}\right)$, $\left(p_{1}, p_{2}\right),\left(p_{2}, p_{1}\right)$, and $\left(p_{2}, p_{2}\right)$ belong to $G$, then arbitrary sequences of $p_{1}$ and $p_{2}$ are trajectories for $G$. This set of trajectories is topologically equivalent to a shift on two symbols. Still, the only points of $G$ visited by these trajectories are the canard pairs above. Thus, there may be large sets of trajectories that visit the same points of $G$, but in a different temporal order. When we examine the singular limit of the van der Pol system, these sets of trajectories are limits of chaotic invariant sets of the return map for a cross-section of the van der Pol system. Therefore, we still regard these sets of trajectories for $G$ as chaotic invariant sets even though they visit only a finite number of points of $G$.

We next describe the symbolic dynamics of the examples presented in the last section. When $(a, \omega)=(1.1,1.57), H$ has a single discontinuity $\theta_{2 s}$ and is strictly increasing. See Figure 4. There are two fixed points of $H$, one stable, denoted $v_{s}$, and one unstable, denoted $v_{u}$. The extensions to $H$ consist of two vertical intervals at the discontinuity, both ending at the maximal canard point, denoted $\theta_{m}$. The interval $\left(\theta_{2 s}, \theta_{m}\right)$ contains $v_{u}$. The interval $\left(v_{u}, \theta_{2 s}\right)$ is forward invariant, all points iterating to $v_{s}$. To obtain the symbolic description of the nonwandering set of the extended $H$, we select four symbols $\{1,2,3,4\}$ that are assigned to the left-hand "increasing" vertical segment of $G$, the right-hand "decreasing" vertical segment of $G$, the interval $\left[\theta_{2 s}, \theta_{m}\right]$, and the interval $\left[v_{u}, \theta_{2 s}\right]$, respectively. The partition has the properties that $H\left(I_{4}\right) \subset I_{4}$ and $I_{1} \cup I_{2} \cup I_{3}$ is a subset of the "images" of $I_{j}$ for $j=1,2,3$. It follows that there is an invariant set $\Lambda$ in $I_{1} \cup I_{2} \cup I_{3}$ that is topologically equivalent to the full shift on three symbols. Note that $H$ is expanding on $I_{3}$. Points that do not remain in $I_{1} \cup I_{2} \cup I_{3}$ converge monotonically to $v_{s}$ once they map into $I_{4}$. We conclude that the nonwandering set of $G$ consists of $\Lambda$ and $v_{s}$.

The second example that we consider has parameter values $(a, \omega)=(4,1.51)$. Figure 5 shows the graph of $H$ with its extensions for these parameter values. There are three points of discontinuity for $H$, and $H$ has a local minimum in one subinterval on which it is continuous and a local maximum in another subinterval, and $H$ is increasing on the third subinterval. There are a pair of canard extensions at each point of discontinuity. We partition $G$ into nine subintervals $I_{1}, \ldots, I_{9}$ with $I_{1}$ the subinterval with a local maximum, $I_{4}$ the subinterval with a local minimum, and $I_{7}$ the subinterval on which $H$ is increasing. For $j=1,4,7$, we set $I_{j+1}$ to be the canard segment at the right endpoint of $I_{j}$. We set $I_{3}$ to be the canard segment at 
the left endpoint of $I_{4}, I_{6}$ to be the canard segment at the left endpoint of $I_{7}$, and $I_{9}$ to be the canard segment at the left endpoint of $I_{1}$.

There are two stable fixed points of this example, one in $I_{1}$ and one in $I_{4}$. All of the points in $I_{1}$ iterate to the fixed point in $I_{1}$, and all of the points in $I_{4}$ iterate to the fixed point in $I_{4}$. The canard extensions $I_{2}$ and $I_{6}$ contain fixed points. The images of the "lower" canard segments $I_{2}, I_{5}$, and $I_{8}$ contain the canard segments $I_{2}, I_{3}$, and points in $I_{1}$ and $I_{4}$ that iterate to the stable fixed points. The images of the "upper" canard segments $I_{3}, I_{6}$, and $I_{9}$ contain the canard segments $I_{5}, I_{6}$ and points in $I_{4}$ and $I_{7}$, including a preimage $p$ of the canard segments $I_{2}$ and $I_{3}$ that lies in $I_{7}$. Points other than $p$ in $I_{7}$ have images in $I_{1}$ or $I_{4}$ and iterate to the fixed points. Looking at these images, we find that there is an invariant set $\Lambda$ topologically equivalent to a subshift with symbols $2,3,5,6,7$ and that the transition matrix $^{1}$ for the subshift is

$$
\left(\begin{array}{lllll}
1 & 1 & 0 & 0 & 0 \\
0 & 0 & 1 & 1 & 1 \\
1 & 1 & 0 & 0 & 0 \\
0 & 0 & 1 & 1 & 1 \\
1 & 1 & 0 & 0 & 0
\end{array}\right),
$$

reflecting that the images of $I_{2}, I_{5}$, and $p$ contain $I_{2} \cup I_{3}$ while the images of $I_{3}$ and $I_{6}$ contain $I_{5} \cup I_{6} \cup\{p\}$. The nonwandering set of $H$ consists of the two stable fixed points and $\Lambda$.

The third example of a structurally stable nonwandering set we display is for parameter values $(a, \omega)=(1.02,1.19)$. Figures 6 and 7 show the graphs of the first and second iterates of the half return map $H$. There are no fixed points of $H$, but the graph of $H^{2}$ resembles the dynamics of $H$ at the parameter values $(a, \omega)=(1.1,1.57)$. There is a stable periodic orbit of period 2 for $H$. There are also two regions containing canards in each of which the extension of $H^{2}$ has an invariant set that is topologically equivalent to a full shift on three symbols. These two invariant sets of $H^{2}$ are interchanged by $H$. Thus $G$ has an invariant set that is topologically equivalent to a subshift with six symbols whose transition matrix is

$$
\left(\begin{array}{ll}
\mathbf{0} & \mathbf{I} \\
\mathbf{I} & \mathbf{0}
\end{array}\right),
$$

where $\mathbf{0}$ is the $3 \times 3$ zero matrix and $\mathbf{I}$ is the $3 \times 3$ matrix with all components 1 .

6. Bifurcations. Bifurcations of the reduced system and its half return map $H$ occur where the system is not structurally stable. A long term goal is to classify the bifurcations that occur as the parameters $a$ and $\omega$ vary, but this paper concentrates on several bifurcations that affect changes in the number of fixed points of $H$ and bifurcations that bound a few parameter regions of structurally stable systems with chaotic invariant sets.

Figure 13 reproduces the bifurcation diagram of fixed points of $H$ without its extension [14]. Recall that in the region where there are folded foci, the bifurcations of fixed points

\footnotetext{
${ }^{1}$ Recall that a subshift of finite type [8] with symbols $s_{1}, \ldots, s_{n}$ and transition matrix $T=\left(T_{i j}\right)($ an $n \times n$ matrix with components 0 and 1$)$ consists of all sequences $\left\{a_{i}\right\}$ with $a_{i} \in\left\{s_{1}, \ldots, s_{n}\right\}$ and $T_{a_{i} a_{i+1}}=1$ for all $i$. One-sided shifts are indexed by $i \geq 0$ while two-sided shifts are indexed by $i \in Z$.
} 


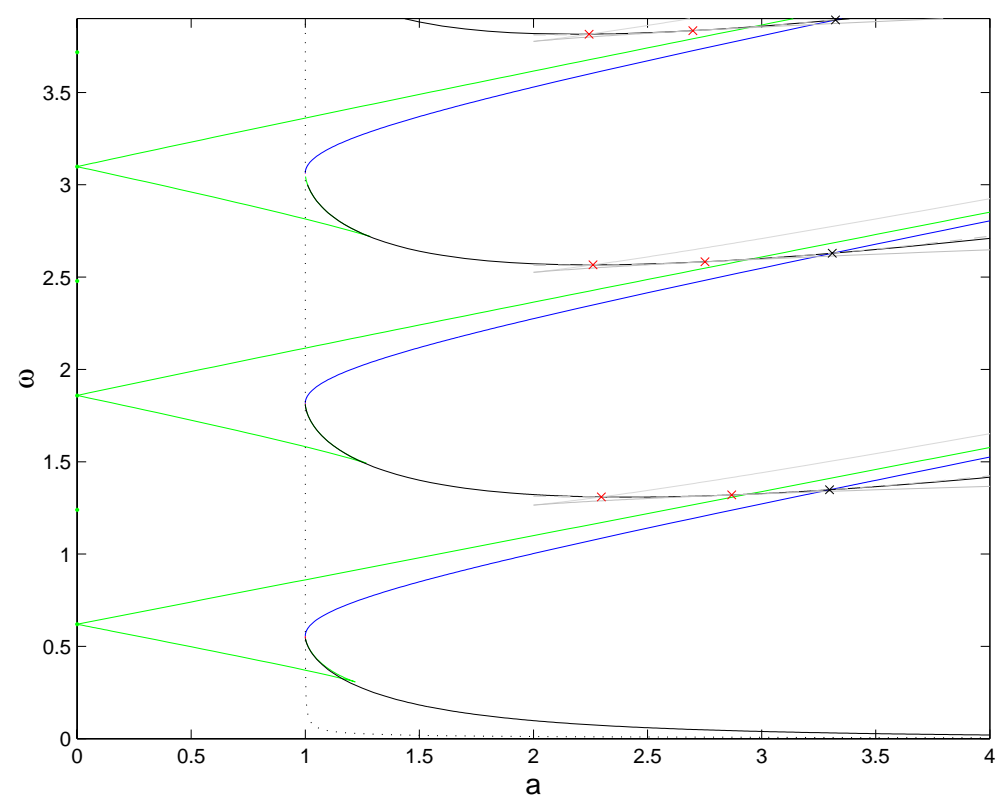

Figure 13. The bifurcation diagram of fixed points of $H$. The green curves are saddle-node bifurcations, the black curves are right homoclinic bifurcations, the blue curves are left homoclinic bifurcations, and the gray curves are points where there is a point of $W_{s}$ tangent to the circle $S_{2}$.

of $H$ that were found are two types of homoclinic orbits (black and blue in the diagram) and saddle-node bifurcations (green). For each circuit number of the corresponding periodic orbits in the reduced system, there is a curve of bifurcations of each of these three types. Discontinuities of $H$ occur at intersections of $W_{s}$, the stable manifold of the folded saddle, with the circle $x=2$ in the phase space, so the number of discontinuities of $H$ changes at points where $W_{s}$ intersects the circle $x=2$ tangentially. The locations of these parameter values are drawn as gray curves in the bifurcation diagram. They play an even more important role in the bifurcations of the extended half return map because each discontinuity gives rise to canards and vertical segments in the graph of the extended $H$. We now turn to the extension of $H$ and its bifurcations.

In the parameter region $a<2$, there are no tangencies of the vector field with the circle $x=2$, so $H$ has no local maxima and minima and just a single discontinuity. In the extension of $H$, the left and right values of the maximal canards are the same. To characterize the dynamics of $H$, we are especially interested in the kneading data, i.e., the itineraries of the critical values. There are two critical values, namely, $\theta_{m}$, the value of the maximal canard, and $\theta_{r}=\theta_{1 u}+1 / 2$, the right-hand limit of $H$ at the discontinuity. The graphs of the extended half return map are limits of piecewise smooth circle maps with two critical points. Such maps can have quasi-periodic trajectories, but they always have periodic orbits as well [5]. The universal bifurcation diagram for smooth circle maps with two critical values is essentially two dimensional. The properties of $G$, the relation associated with the extension of $H$, will be similar to those of these circle maps in the parameter region $1<a<2$ with folded foci. We look for bifurcations of $G$ at locations where the itineraries of the two critical values do 


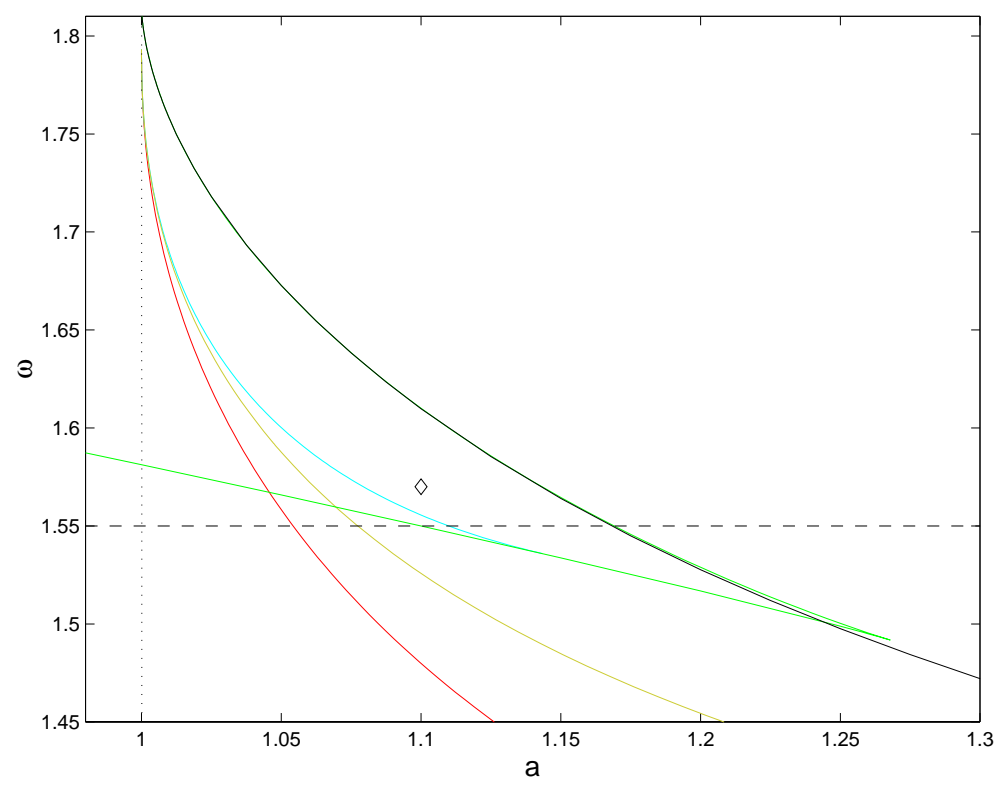

Figure 14. A detail from the bifurcation diagram. The diamond is the point $(a, \omega)=(1.1,1.57)$. In this figure, we have added an additional bifurcation curve. The yellow curve is the set of parameter values where $H\left(\theta_{m}\right)=\theta_{2 s}$.

not iterate to stable periodic orbits. We begin by looking at the boundary of the region of structurally stable systems in parameter space around $(a, \omega)=(1.1,1.57)$.

In the previous section, we showed that when $(a, \omega)=(1.1,1.57)$, the nonwandering set of $G$ consists of a fixed point and an invariant set $\Lambda$ topologically equivalent to a full shift on three symbols. We discuss here the bifurcations that limit the parameter region in which $G$ has a nonwandering set topologically equivalent to this one. The following properties of $H$ and its extension imply that there is a hyperbolic invariant set $\Lambda$ equivalent to the shift on three symbols. We first summarize the relevant properties of $H$ :

- $H$ has a single jump discontinuity at $\theta=\theta_{2 s}$ with $\theta_{r}<\theta_{2 s}$;

- $H$ is continuous on $S^{1}-\left\{\theta_{2 s}\right\}$;

- $H$ has only two fixed points $v_{u}$ and $v_{s}$, with $\theta_{2 s}<v_{u}<v_{s}$, where $v_{u}$ is unstable and $v_{s}$ is stable;

- and $\theta_{m} \geq v_{u}$.

As described in the previous section, the nonwandering set of the extension $G$ of $H$ consists of just the stable fixed point and $\Lambda$.

Figure 14 displays the curve $\mu$ in cyan along which $\theta_{m}=v_{u}$. There is a sequence of bifurcations that accumulate on $\mu$ from below (i.e., smaller $\omega$ ). Below $\mu$, the nonwandering set of $G$ no longer contains an invariant set equivalent to a shift on three symbols. If $\theta_{2 s}^{-}$and $\theta_{2 s}^{+}$denote the two adjacent points in $X$ where the vertical segments are added, $\left(\theta_{2 s}^{-}, \theta_{2 s}^{-}\right)$, $\left(\theta_{2 s}^{-}, \theta_{2 s}^{+}\right),\left(\theta_{2 s}^{+}, \theta_{2 s}^{-}\right)$, and $\left(\theta_{2 s}^{+}, \theta_{2 s}^{+}\right)$are all in $G$ as long as $\theta_{m}>\theta_{2 s}$. As mentioned earlier, this means that arbitrary sequences of $\theta_{2 s}^{-}$and $\theta_{2 s}^{+}$are trajectories of $G$; this set of trajectories is topologically equivalent to a shift on two symbols, so the nonwandering set of $G$ (minus 


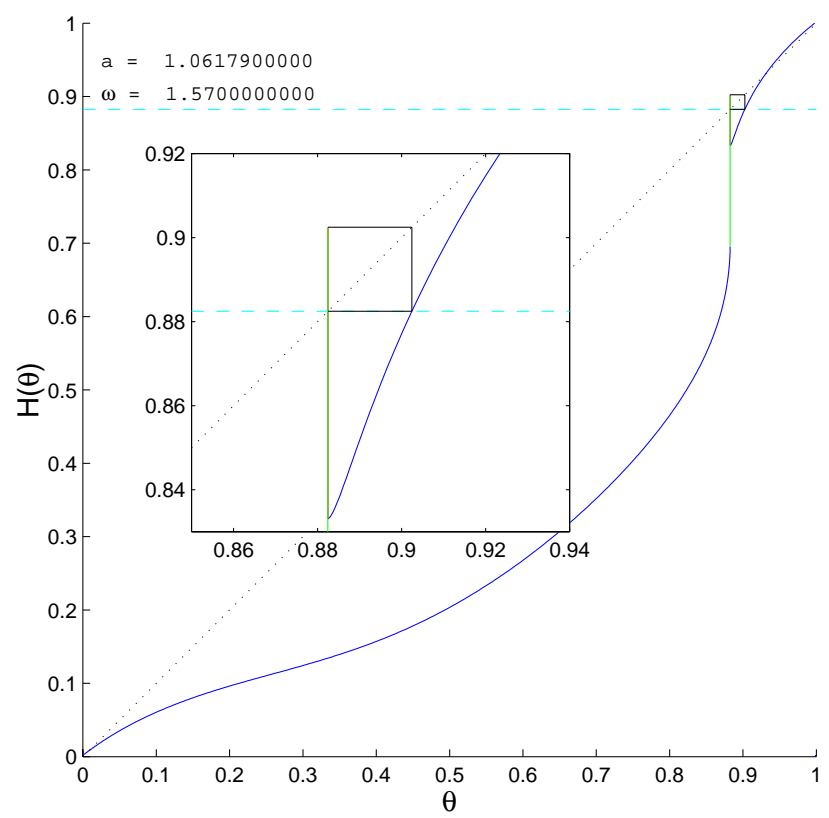

Figure 15. An example of Proposition 6.1 where $c_{1}=\theta_{m}$.

the stable fixed point) contains a shift on two symbols and is properly contained in the shift on three symbols. We give a more precise description of these nonwandering sets. Let $c_{k}=H^{-k}\left(\theta_{2 s}\right)$. We observe that $\lim _{k \rightarrow \infty} c_{k}=v_{u}$.

Proposition 6.1. Let $H, G$, and $\left\{c_{k}\right\}_{k=1}^{\infty}$ be as defined above. Assume that none of the $c_{k}$ are $\theta_{m}$. Let $\left\{c_{1}, c_{2}, \ldots, c_{n}\right\}$ be the set for which $c_{k}<\theta_{m}$. Then the nonwandering set of $G$ consists of the two fixed points of $H$ and a hyperbolic invariant set $\Lambda$ with $n+2$ symbols $\left\{\theta_{2 s}^{-}, \theta_{2 s}^{+}, c_{1}, c_{2}, \ldots, c_{n}\right\}$ and transition matrix

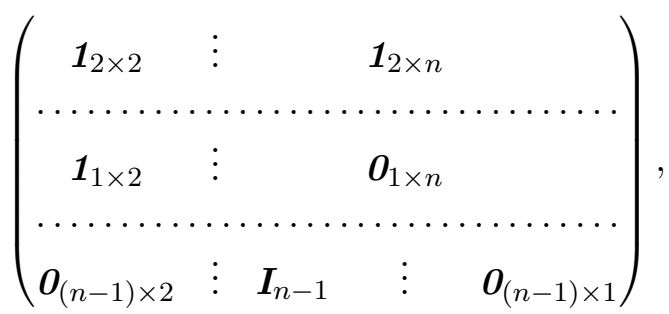

where $\boldsymbol{1}_{m \times n}$ is the $m \times n$ matrix of ones, $\boldsymbol{O}_{m \times n}$ is the $m \times n$ matrix of zeros, and $\boldsymbol{I}_{n}$ is the $n \times n$ identity matrix.

The proposition is proved by observing that the points $\left(\theta_{2 s}^{-}, c_{k}\right)$ and $\left(\theta_{2 s}^{+}, c_{k}\right)$ are in $G$ precisely when $k \leq n$. From either of these points, the subsequent trajectory is $c_{k}, c_{k-1}, \ldots, c_{1}$. By the construction of $G, c_{1}$ can be followed by either $\theta_{2 s}^{-}$or $\theta_{2 s}^{+}$. Note that if $c_{1}>\theta_{m}$, then $\Lambda=\left\{\theta_{2 s}^{-}, \theta_{2 s}^{+}\right\}$, and on $\Lambda, G$ is equivalent to a shift on two symbols; the unextended regions do not play a role in the chaotic invariant sets.

The changes in the structure of the symbolic dynamics occur when $c_{k}=\theta_{m}$ for some $k$; at these points, the kneading data associated with $\theta_{m}$ change. For example, Figure 15 shows 


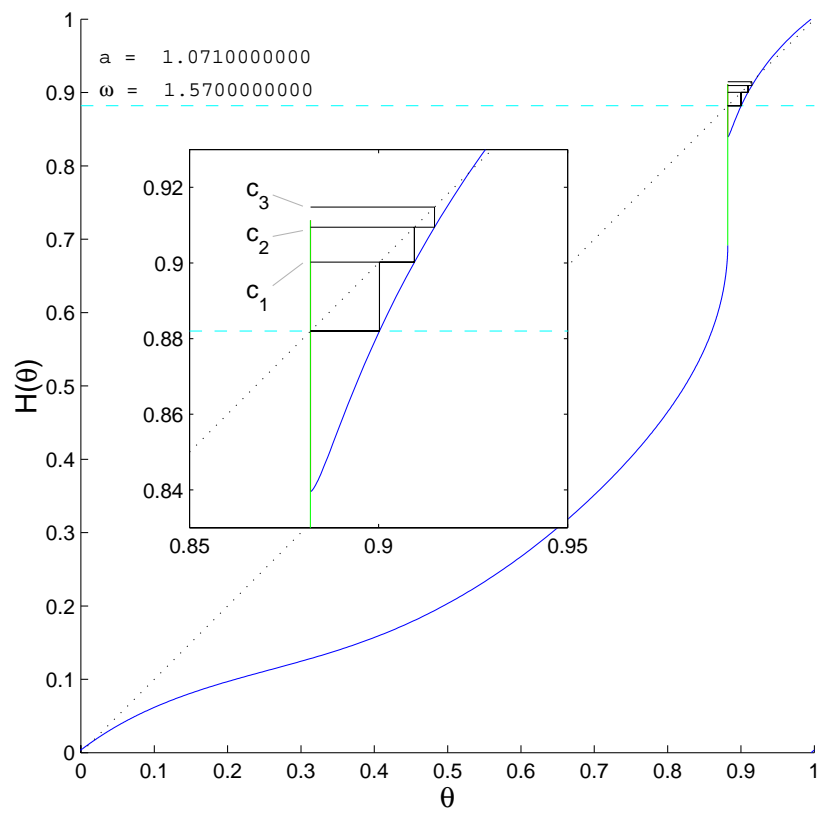

Figure 16. An example of Proposition 6.1 where $c_{2}<\theta_{m}$ but $c_{3}>\theta_{m}$.

a case where $c_{1}=\theta_{m}$. This is a point from the yellow curve in Figure 14. On one side of this curve, the chaotic dynamics are equivalent to a shift on $\left\{\theta_{2 s}^{-}, \theta_{2 s}^{+}\right\}$. On the other side, the symbols are $\left\{\theta_{2 s}^{-}, \theta_{2 s}^{+}, c_{1}\right\}$, and the transition matrix is

$$
\left(\begin{array}{lll}
1 & 1 & 1 \\
1 & 1 & 1 \\
1 & 1 & 0
\end{array}\right)
$$

If we continue to change the parameters in this direction, more bifurcations will occur. For example, Figure 16 shows a case where $c_{2}<\theta_{m}$ but $c_{3}>\theta_{m}$. In this case $\Lambda=\left\{\theta_{2 s}^{-}, \theta_{2 s}^{+}, c_{1}, c_{2}\right\}$, and the transition matrix is

$$
\left(\begin{array}{llll}
1 & 1 & 1 & 1 \\
1 & 1 & 1 & 1 \\
1 & 1 & 0 & 0 \\
0 & 0 & 1 & 0
\end{array}\right) .
$$

Let $\mathcal{U}$ be the connected region in the $(a, \omega)$ plane containing $(1.1,1.57)$ where $G$ has a chaotic invariant set; see Figure 14. There are four curves bounding the region. On the left (near $a=1$ ), we have the curve that marks the transition from a folded node to a folded focus (the black dotted line). The upper boundary is the curve of right homoclinic points (the black solid line). At these points, $\theta_{r}=\theta_{2 s}$; i.e., the right side of the discontinuity is a fixed point. Above this curve there is a thin sliver in which the right-hand vertical extension no longer contains a fixed point, and there is a new stable fixed point just to the right of $\theta_{2 s}$. The upper boundary of the sliver is a min saddle-node curve (the upper green line), where the 
"new" stable fixed point coalesces with $v_{u}$, leaving a single stable fixed point. When the new stable fixed point exists, the preimage of $\theta_{2 s}$ is only a single point that lies in $I_{2}$, the left-hand canard segment of $G$. This fixed point in $I_{2}$ is the only point that maps into the canards for $G$. Consequently, the nonwandering set consists entirely of fixed points - two stable fixed points and two unstable fixed points, one of which lies in $I_{2}$. The two other curves that bound $\mathcal{U}$ are the curve where $\theta_{m}=\theta_{2 s}$ (the red curve) and the max saddle-node curve (the lower green curve). After leaving $\mathcal{U}$ by crossing the red curve, $\theta_{m}<\theta_{2 s}$, and there are no fixed points in the vertical extensions. The green curve is the saddle-node curve, and just below this curve, $H$ does not have any fixed points.

Two additional bifurcation curves in $\mathcal{U}$ that divide parameter regions with topologically inequivalent nonwandering sets are plotted. The curve where $c_{1}=\theta_{m}$ is plotted in yellow, and the curve where $v_{u}=\theta_{m}$ is plotted in cyan. Thus the region bounded by the yellow, lower green, red, and black dotted curves gives the set of parameters where $c_{1}>\theta_{m}$. The region bounded by the solid black, lower green, cyan, and black dotted lines gives the set of parameters where $v_{u}<\theta_{m}$, so the dynamics on $\Lambda$ can be described as a full shift on three symbols. The infinite sequence of bifurcation curves discussed above lies between the yellow and cyan lines. There is one curve for each equation $c_{n}=\theta_{m}$. They are not shown in Figure 14, but they converge to the cyan line as $n$ increases.

This example illustrates some of the new types of bifurcations arising from canards in the extension $G$ of the half return map $H$. In seeking to classify the different types of bifurcations, we note that extended maps $G$ which are not at bifurcation parameters have the property that their critical values tend to stable periodic orbits. Limit sets for the critical values that are not stable periodic orbits can be unstable periodic orbits, subsets of chaotic invariant sets, or quasi-periodic trajectories. There are also parameters at which the number of discontinuities change and where the maximal canards of the reduced system move from the section $S_{1}$ to the section $S_{-1}$. This plethora of possibilities is sufficiently daunting that we do not attempt to determine here the types or locations of more than a few bifurcations of $G$. We focus upon those whose defining equations are expressed in terms of $G$ itself rather than higher iterates.

One of the main qualitative differences in canards of the reduced systems is in the location of maximal canards. When $a$ is large enough and large enough relative to $\omega$, oscillations of the reduced system induced by the forcing are large enough that the saddle separatrix $\gamma_{u}$ intersects the circle $S_{-1}$. The transition between parameter values for which $\gamma_{u}$ intersects the circle $S_{-1}$ and those for which it does not occur when there are heteroclinic orbits that connect the folded saddles on $S_{1}$ and $S_{-1}$. The parameter values with these heteroclinic orbits divide the parameter space into two regions that we call $M_{1}$ and $M_{-1} . M_{1}$ lies above the heteroclinic curve in the $(a, \omega)$ plane and yields maximal canards on the circles $S_{1}$. $M_{-1}$ lies below the heteroclinic curve in the $(a, \omega)$ plane and yields maximal canards on the circles $S_{-1}$. In $M_{1}$, the maximal canard value associated with the jump back canards (i.e., those that jump to $x>1$ ) is $\theta_{m}$. The maximal canard value associated with the jump away canards (i.e., those that jump to $x<-1$ ) is either $\theta_{m}+0.5$ (when $\left.x^{\prime}\left(\theta_{m},-2\right)>0\right)$ or $\theta_{m 2}+0.5$, where $\left(\theta_{m 2},-2\right)$ is the previous point on the slow flow trajectory through $\left(\theta_{m},-2\right)$ (when $x^{\prime}\left(\theta_{m},-2\right)<0$ ). Note that $H\left(\theta_{m}\right)=H\left(\theta_{m 2}\right)$ in the second case. Though similar methods can be used to analyze bifurcations in $M_{-1}$, this paper considers only bifurcations of canards in the region $M_{1}$.

Among the new bifurcation types that we expect from the extensions of $H$ are parameters 


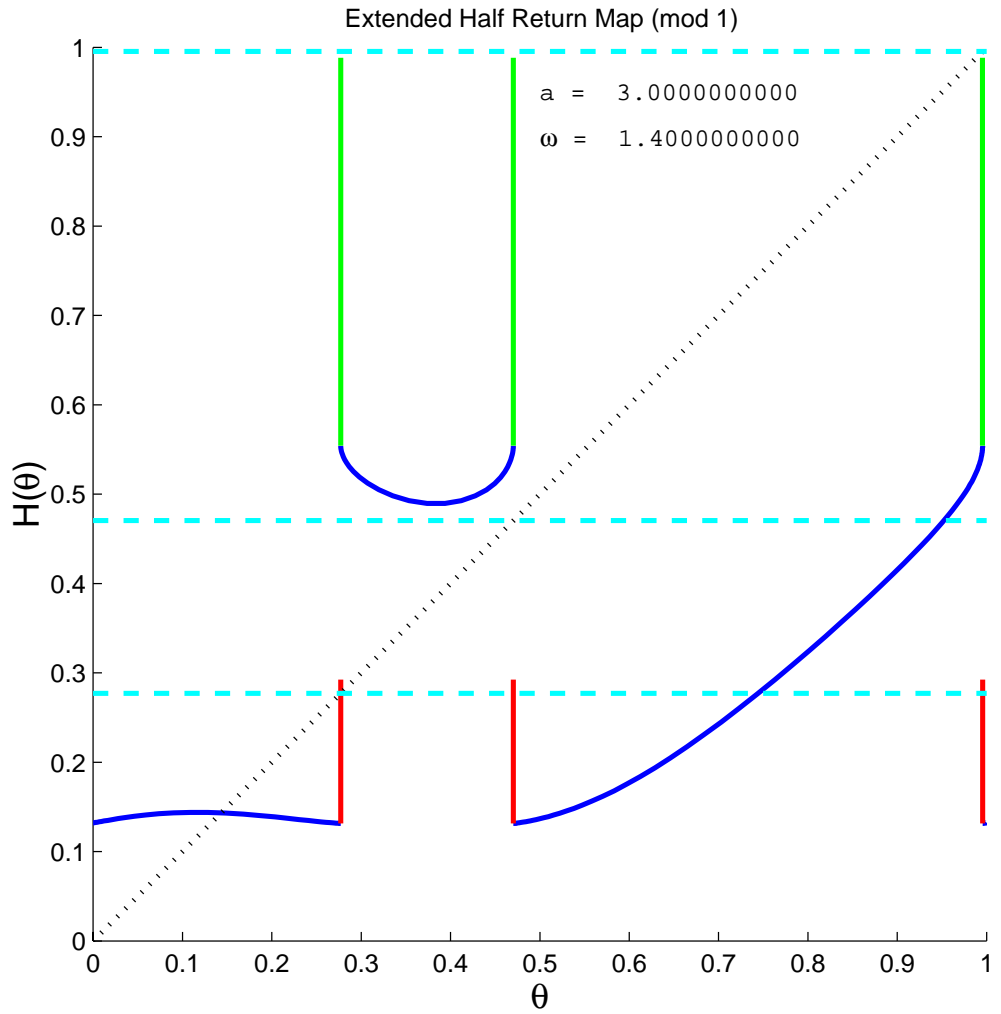

Figure 17. A plot of the extended half return map for parameter values $(a, \omega)=(3,1.4)$.

where $H\left(\theta_{m}\right)$ is a critical point or unstable fixed point; specifically,

1. $H\left(\theta_{m}\right)$ is a discontinuity point, creating a "maximal canard fixed point," or

2. $H\left(\theta_{m}\right)=q$ with $q$ an unstable fixed point of $H$ (not in a canard), or

3. $H\left(\theta_{m}\right)=\arcsin (2 / a)$, the local minimum or maximum of $H$.

If both maximal canard values are $\theta_{m}+0.5$, the first of these bifurcations is at the boundary of existence of a chaotic invariant set of trajectories of $G$ that is topologically equivalent to the shift on two symbols. This type of bifurcation cannot occur in families of smooth maps of the circle or interval. In smooth maps, shifts on two symbols are created from maps without chaotic dynamics by an infinite set of bifurcations with intervening parameter intervals in which the critical point tends to stable periodic orbits [11].

Where the maximal canard values differ, it is less apparent what changes in the nonwandering set occur at a bifurcation with a maximal canard fixed point. Figures 17, 18, and 19 show graphs of the extended half return map for $a=3$ and $\omega=1.4,1.409,1.43$, respectively. The value $\omega=1.409$ is close to a maximal canard fixed point, but the most visible effect of the bifurcation in the graph of $H$ is to switch which canard branch of $H$ contains the fixed point. The constraint that $H$ have the same image at both maximal canard values prevents the simultaneous existence of two fixed points within canards near these parameter values.

We have seen in the examples with $a=1.1$ that bifurcations in which a maximal canard value maps into a fixed point can change the structure of chaotic invariant sets without 


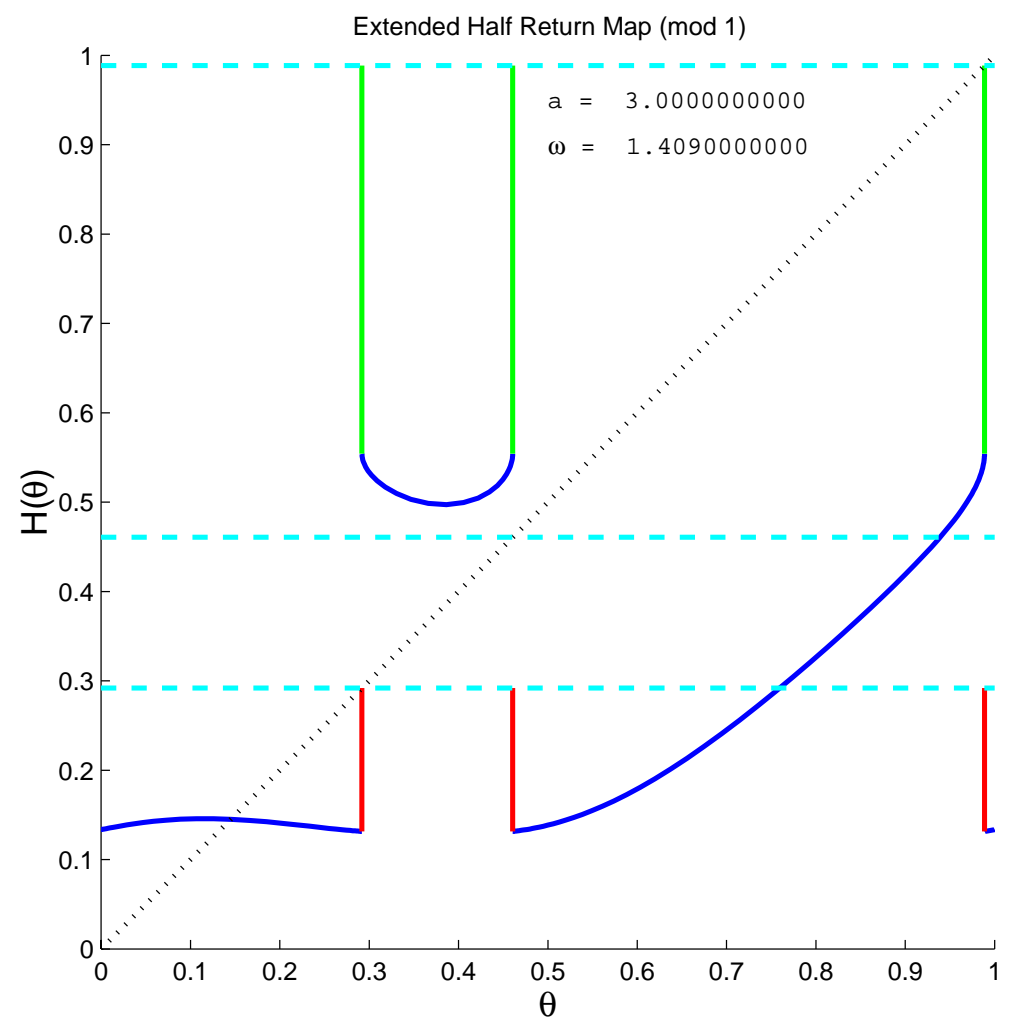

Figure 18. A plot of the extended half return map for the parameter values $(a, \omega)=(3,1.409)$.

changing the number of fixed points of $G$. The bifurcations in which maximal canard values map into tangency points $\theta=\arcsin (-2 / a) /(2 \pi)$ do not seem to affect the number of fixed points of $H$. Instead, these bifurcations are associated with changes in the qualitative structure of the canard extensions of $H$; there is a transition from parameters at which the two maximal canard values are equal to parameters at which these values differ. The transition occurs when the maximal canard maps into the larger value of the tangency point $\theta=\arcsin (-2 / a) /(2 \pi)$, as described in section 3 .

There are parameter regions where the nonwandering set of $G$ contains an invariant set on which $G$ is equivalent to a subshift of finite type. We have already seen examples, in particular, the region $\mathcal{U}$ in which Proposition 6.1 applies. Here we identify additional regions where this is true, but we do not attempt to completely characterize the nonwandering set as we did in Proposition 6.1. The regions that we consider are those where there are two fixed points in the canard extensions. We have observed several qualitative cases where this occurs.

The example $(a, \omega)=(1.1,1.57)$ shows a case where the left and right vertical extensions are adjacent and have a fixed point. In this case, the nonwandering set has an invariant subset $\left\{\theta_{2 s}^{-}, \theta_{2 s}^{+}\right\}$on which trajectories are topologically equivalent to a shift on two symbols. The parameter region where this condition holds is bounded above by the black curve of right homoclinic points and bounded below by the red curve of maximal canard fixed points.

There is a region adjacent to the previous region, bounded by the red curve of maximal 


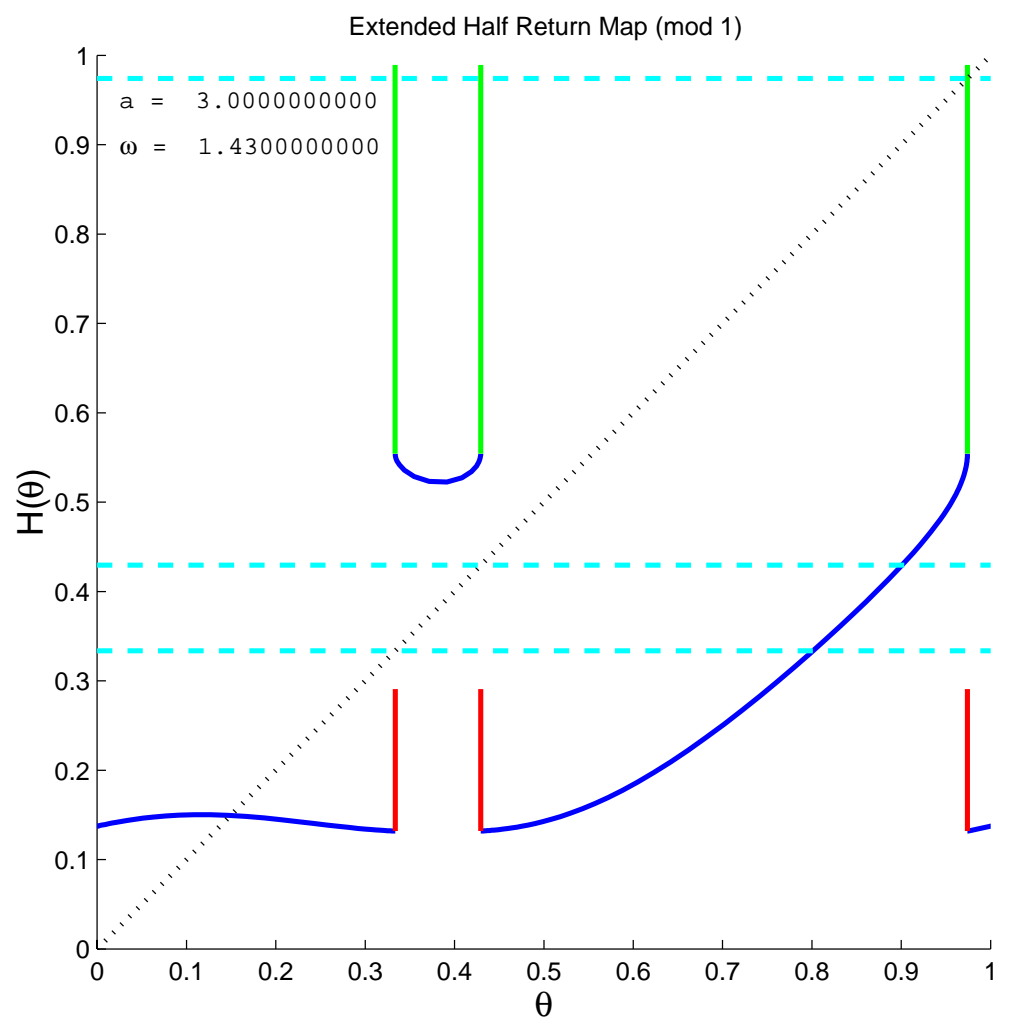

Figure 19. A plot of the extended half return map for the parameter values $(a, \omega)=(3,1.43)$.

canard fixed points, the black curve of right homoclinics, and the gray curve where $W_{s}$ is tangent to $S_{2}$, in which there are two different jump back canard extensions with fixed points. This region is the roughly triangular-shaped region shown in the lower right inset in Figure 23. An example is $(a, \omega)=(2.4,1.3)$ (see Figure 20). Let $d_{1}, d_{2}$, and $d_{3}$ be the $\theta$ coordinates of the discontinuities, and let $d_{i}^{-}$and $d_{i}^{+}$be the left and right adjacent points in $X$ corresponding to these discontinuities. Then there are trajectories made up of only $\left\{d_{1}^{+}, d_{2}^{-}\right\}$for which $G$ is topologically equivalent to a shift on two symbols.

There is a region where a jump back canard extension and a jump away canard extension have fixed points at different discontinuities. This occurs in the example $(a, \omega)=(4,1.51)$; see Figure 2. Define $d_{i}^{ \pm}$as before. In this case, there are trajectories made up of $\left\{d_{1}^{-}, d_{1}^{+}, d_{2}^{-}, d_{2}^{+}\right\}$ for which $G$ is topologically equivalent to a subshift with the transition matrix

$$
\left(\begin{array}{llll}
1 & 1 & 0 & 0 \\
0 & 0 & 1 & 1 \\
1 & 1 & 0 & 0 \\
0 & 0 & 1 & 1
\end{array}\right) .
$$

(Note that this transition matrix is a submatrix of the transition matrix derived in the example where $(a, \omega)=(4,1.51)$.) The region in the $(a, \omega)$ plane where this condition holds that also contains $(4,1.51)$ is bounded below by the black right homoclinic curve and above by the blue 


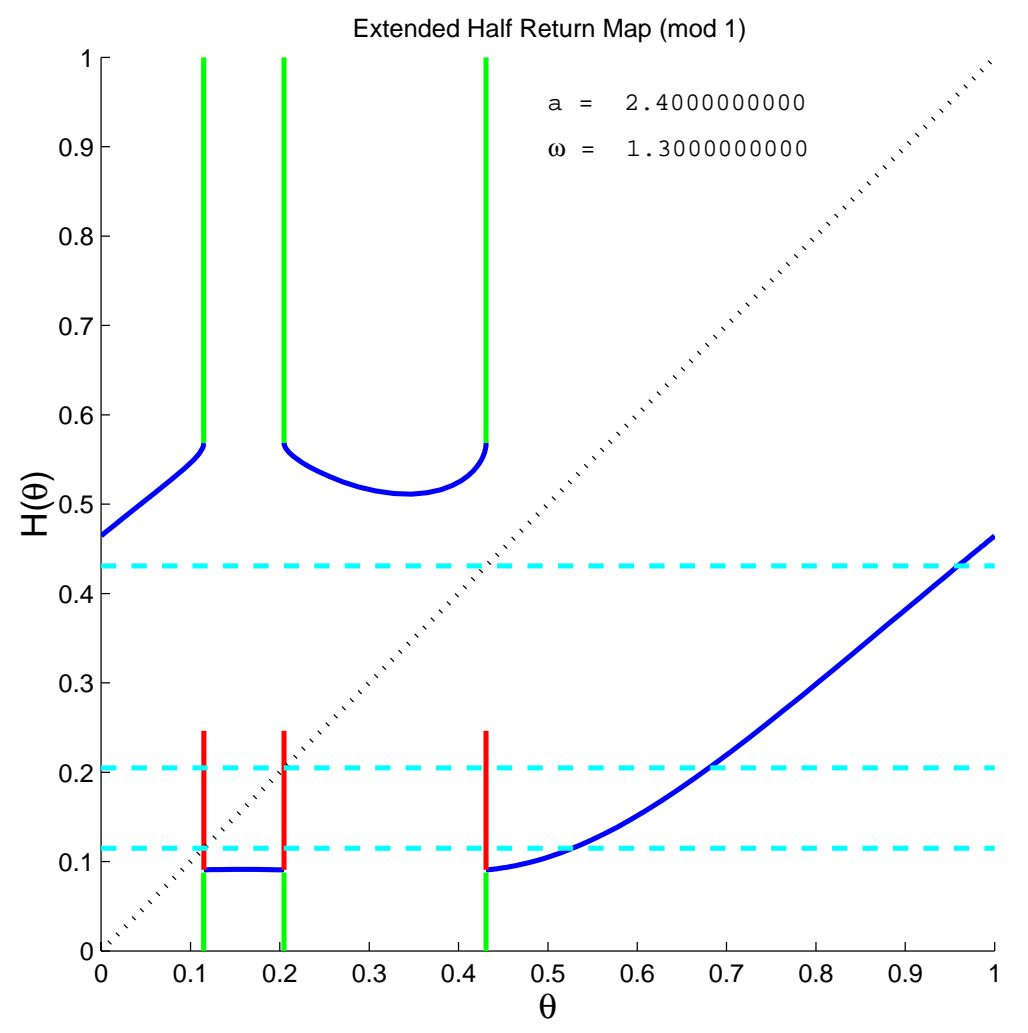

Figure 20. An example where two different jump back canard extensions have fixed points; $(a, \omega)=(2.4,1.3)$.

left homoclinic curve (see Figure 22 or Figure 23). This region appears to be unbounded as $a$ increases.

There are regions where two different jump away canard extensions have fixed points. An example is $(a, \omega)=(6.5,3.41)$; see Figure 21. As in the case where two different jump back canards have fixed points, on the set $\left\{d_{2}^{-}, d_{3}^{-}\right\}, G$ is topologically equivalent to a shift on two symbols. The region where this occurs is bounded below by the red curve of maximal canard fixed points and above by the blue curve of left homoclinic points. We have observed that for circuit number 1, the curve of maximal canard fixed points does not intersect the curve of left homoclinic points, so the case where two different jump away extensions have fixed points occurs only in regions associated with circuit number 2 or higher.

Figure 22 is an augmented bifurcation diagram in which we have added bifurcation curves for the three types of bifurcations involving maximal canard points discussed above. Curves of parameters with maximal canard fixed points are drawn red, curves of parameters with maximal canard points mapping to fixed points are drawn cyan, and curves of parameters with maximal canard points mapping to points of tangency of the slow flow with $S_{-2}$ are drawn yellow. In addition, we draw the parameter curve on which there are heteroclinic connections between the two folded saddles of the reduced system in magenta.

Thus far, we have investigated an extended half return map for the reduced system, seeking to understand the role of canard solutions in the full solution set to the forced van der Pol 


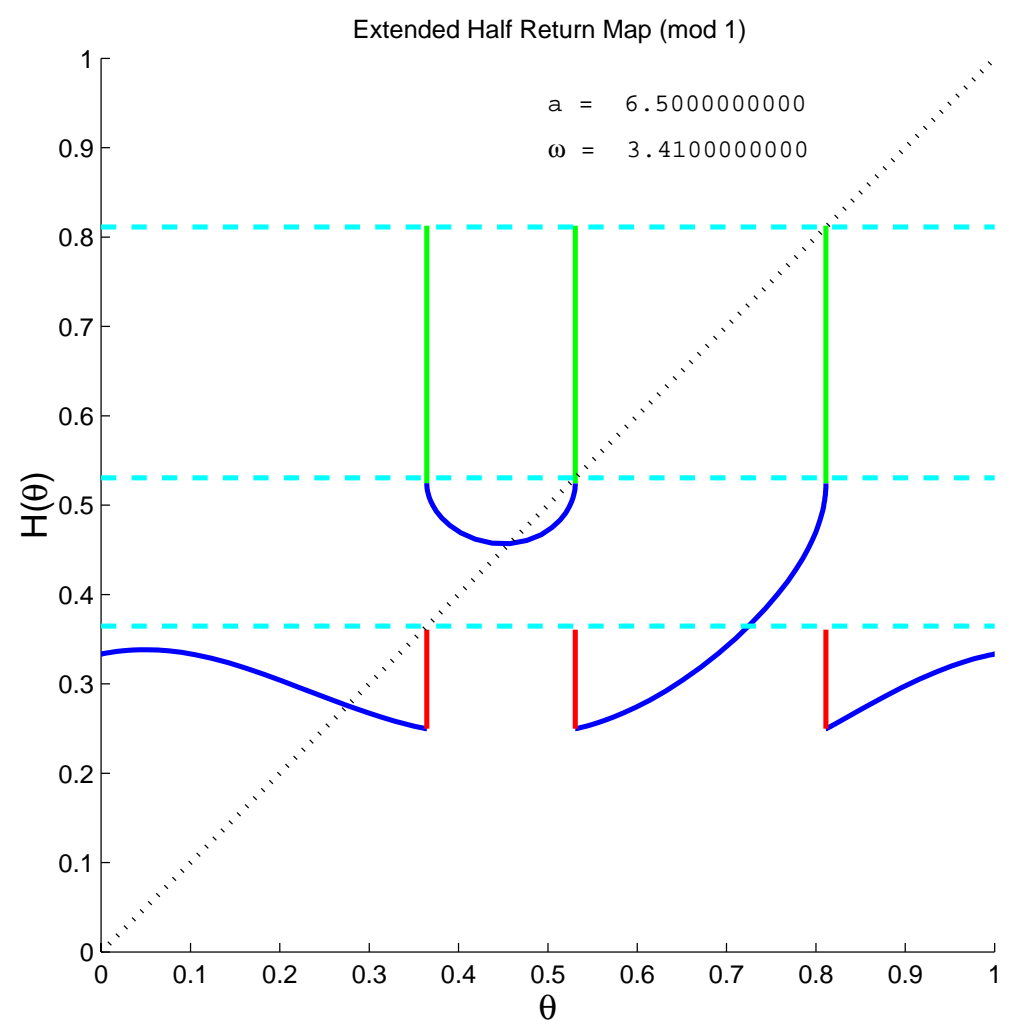

Figure 21. An example where two different jump away canard extensions have fixed points; $(a, \omega)=$ $(6.5,3.41)$.

equation. In particular, we have shown that the canard extensions to the reduced system play an important role in the bifurcations that occur in that system. In the next section, we explore the relationship between the reduced system with its canard extensions and the full system using the half Poincaré map and the singular half Poincaré map. The concept of candidates [4] is useful in formalizing this relationship. Candidates are continuous curves consisting of the concatenation of trajectory segments, the slow flow on the stable sheets of the critical manifold, trajectory segments of the slow flow on the unstable sheets of the critical manifold (canards), and segments parallel to the $\theta$ axis with the orientation of the fast flow. All of the possible candidates are represented by orbits of our singular half Poincaré map. However, it is not evident that all the candidates are limits of trajectories in the full system as $\varepsilon \rightarrow 0$. Though we do not attempt to prove that all candidates are limits of trajectories in the full system, we present numerical computations in the next section that provide evidence that they are.

7. Comparisons of the reduced and full systems: Numerical calculations with $\varepsilon>0$. The reduced system is a means of understanding the full van der Pol system (1.1) with $\varepsilon \neq 0$. In this section, we consider the relationship between the full system and the reduced system by comparing computations of Smale horseshoes [22] and bifurcations in the reduced system 


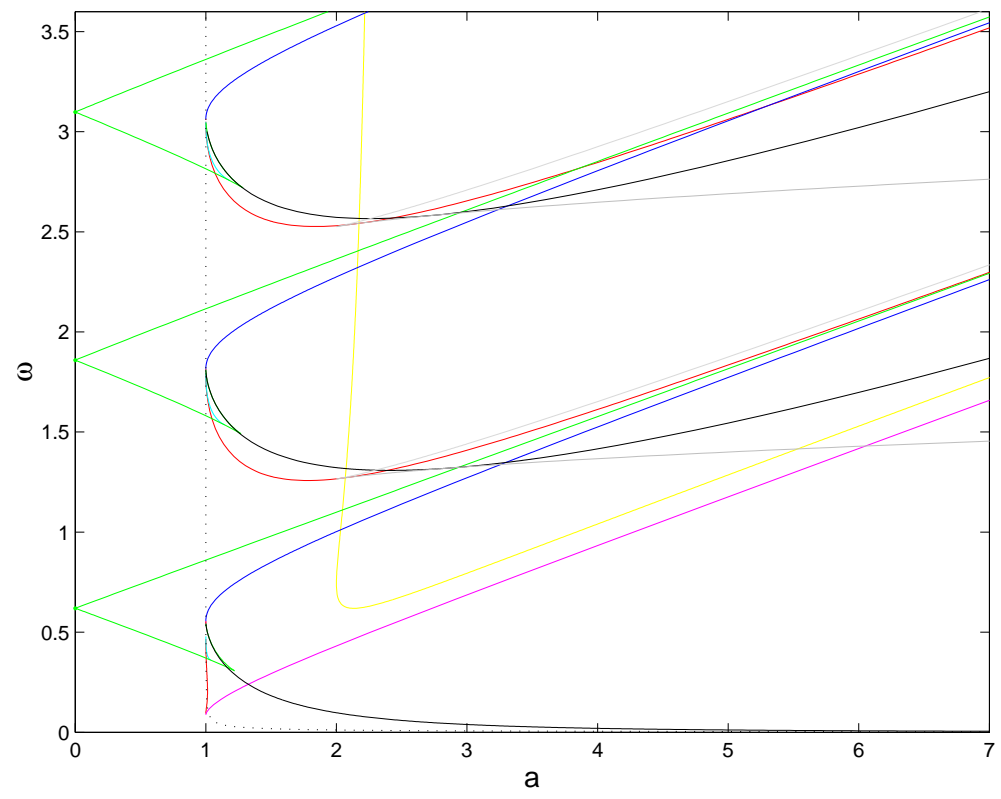

Figure 22. The bifurcation diagram of fixed points of $G$, the canard extension of $H$. The red curves give the location of maximal canard fixed points. The magenta curve shows the location of heteroclinic connections between the two folded saddles of the reduced system. The cyan curves (better seen in Figure 23) show parameters where maximal canard points map to fixed points and the yellow curves show parameters where maximal canard points map to points of tangency of the slow flow with $S_{-2}$.

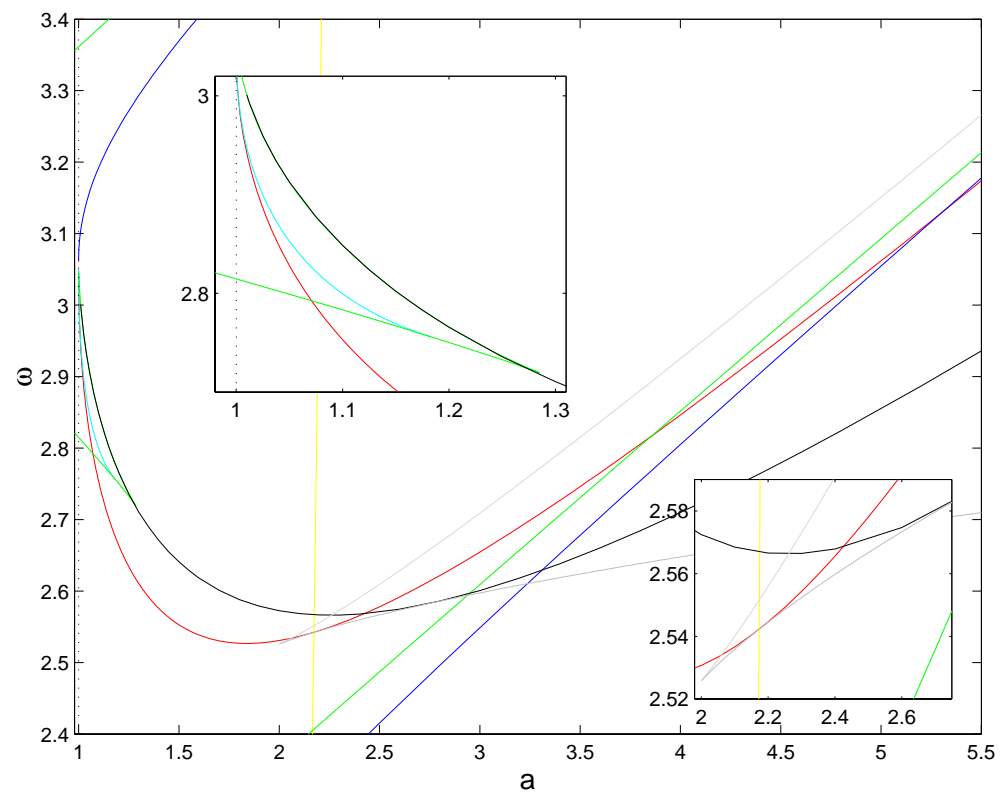

Figure 23. A closer look at parts of the bifurcation diagram of fixed points of $G$ shown in Figure 22. 


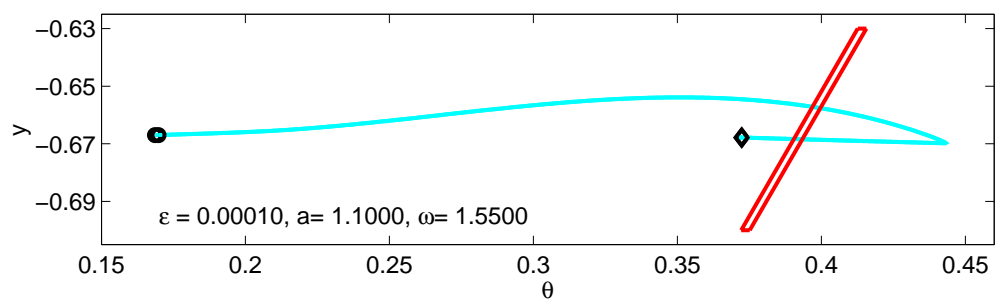

Figure 24. A Poincaré map that exhibits a horseshoe in (1.1). The parameter values are $\varepsilon=10^{-4}, a=1.1$, and $\omega=1.55$. The cyan curves are the images of the horizontal segments at the end of the region plotted in red. Because of the strong contraction on the stable slow manifold, the two cyan curves are indistinguishable in this plot. The ends of the cyan curves are marked with circles on the left and diamonds on the right.

to computations of the full system with similar parameter values. The results demonstrate that our analysis of canards in the reduced system gives a reliable guide to dynamical behavior associated with canards in the full system.

Numerical computation of trajectories with canards is difficult with initial value solvers [15]. When $\varepsilon>0$ is small, the exponential growth of deviations from an unstable sheet of the slow manifold is sufficiently rapid that the round-off error in representing an initial point on the slow manifold will give a trajectory that has a jump point close to the initial point. This is an unescapable feature of slow-fast dynamical systems. The use of different initial value solvers, even "stiff" solvers, does not resolve the difficulty. This does not make it hopeless to compute approximations to trajectories with canards, however. Boundary value solvers compute curves that approximately solve a system of differential equations with specified boundary conditions. Their convergence properties are not directly related to the stiffness of the system being solved. We used the program AUTO [9] as a boundary value solver to explore the dynamics of the forced van der Pol system (1.1) for small $\varepsilon>0$. Our goal was to confirm predictions arising from our analysis of canards in the reduced system and its half return map. This is done with two different types of AUTO computations.

The first AUTO computation seeks a Smale horseshoe in the return map $\Phi_{\varepsilon}$. The cyan curves in Figure 24 are the images under the Poincaré map $\Phi_{\varepsilon}$ of the short horizontal line segments at the upper and lower ends of the red parallelogram. The red parallelogram was chosen to include a piece of the strip $\kappa_{\varepsilon}$. There are two cyan curves, but the strong contraction on the stable slow manifold makes the resulting curves indistinguishable in this plot. More examples are shown in Figure 25. These examples illustrate the extreme contraction that is associated with the two time scales in the forced van der Pol system. The expansion of horizontal segments within $\kappa_{\varepsilon}$ by a factor of approximately 50 along the images is also evident in these figures. The combination of the contraction along the long direction of the parallelogram, the expansion along the horizontal direction (resulting from the formation of canards), and the fold that occurs at the maximal canard gives the cyan curves their shape. When $\Phi_{\varepsilon}\left(\kappa_{\varepsilon}\right)$ intersects $\kappa_{\varepsilon}$ as in Figures 24 and 25, we see that $\Phi_{\varepsilon}$ possesses all the ingredients for the existence of a Horseshoe map with a hyperbolic invariant set.

Singular Poincaré maps for parameter values close to those in Figures 24 and 25 are shown in Figure 26. In particular, Figure 26(b) shows the same values of $a$ and $\omega$ as Figure 24, and indeed, the pieces of the plot shown in Figure 24 appear to be well approximated by the 

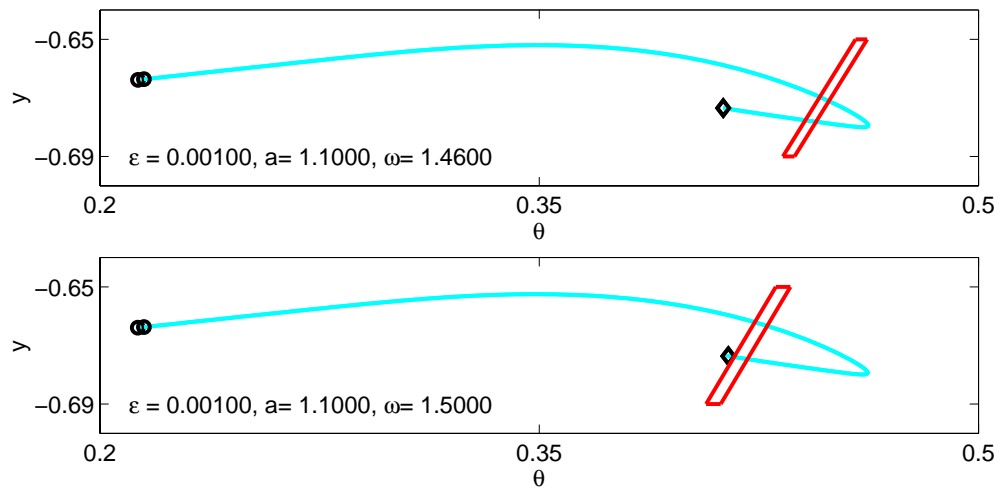

Figure 25. Poincaré maps that exhibit a horseshoe in (1.1). The parameter values are $\varepsilon=10^{-3}, a=1.1$, and (a) $\omega=1.46$, (b) $\omega=1.5$. The cyan curves are the images of the horizontal red line segments under the Poincaré map $\Phi$. Because of the strong contraction on the stable slow manifold, the two cyan curves are indistinguishable in this plot. The ends of the cyan curves are marked with circles on the left and diamonds on the right. (Note that $\varepsilon$ is ten times larger here than in Figure 24. In this case, $\varepsilon$ is large enough that this map was successfully computed with a standard initial value problem solver in MATLAB.)
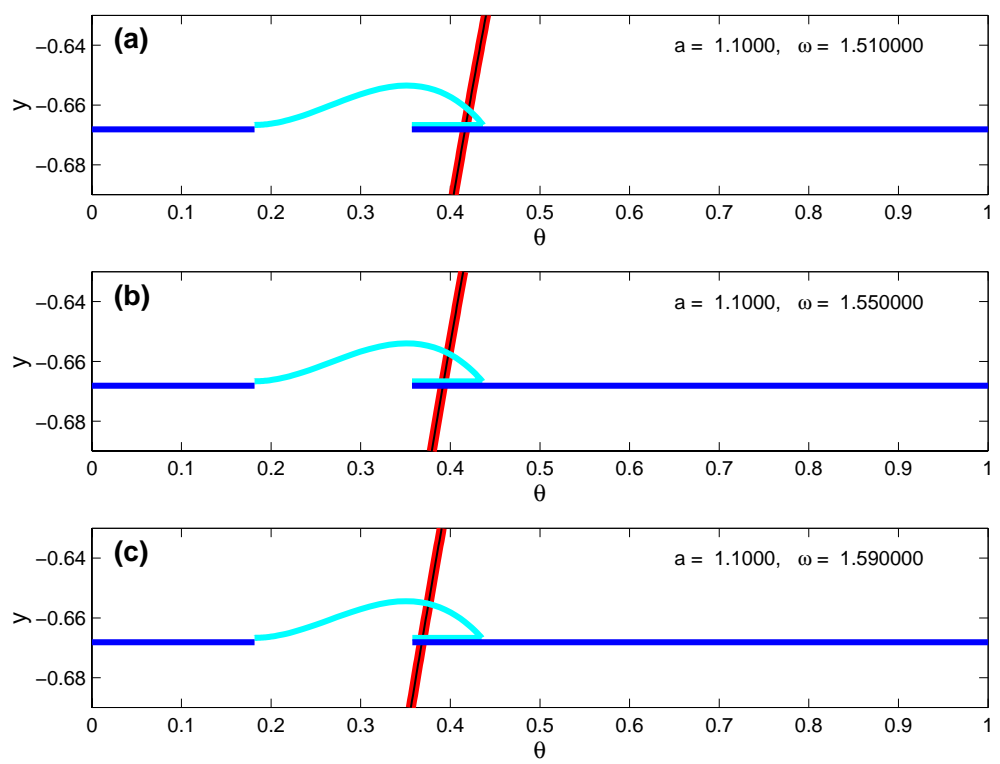

Figure 26. Singular Poincaré maps with $a=1.1$ and several values of $\omega$.

singular map shown in Figure 26. This is evidence of the validity of our representation of canards in the reduced system.

We used the continuation capabilities of AUTO to track families of periodic orbits along a line in the parameter space with $\varepsilon=10^{-4}$ and $\omega=1.55$ with varying $a$ near 1.1. Our goal is to compare the observed bifurcations of the periodic orbits to the bifurcation diagram for the reduced system displayed in Figure 22. Some of the bifurcations of the van der Pol system (1.1) have different types from the corresponding types in the reduced system due to 


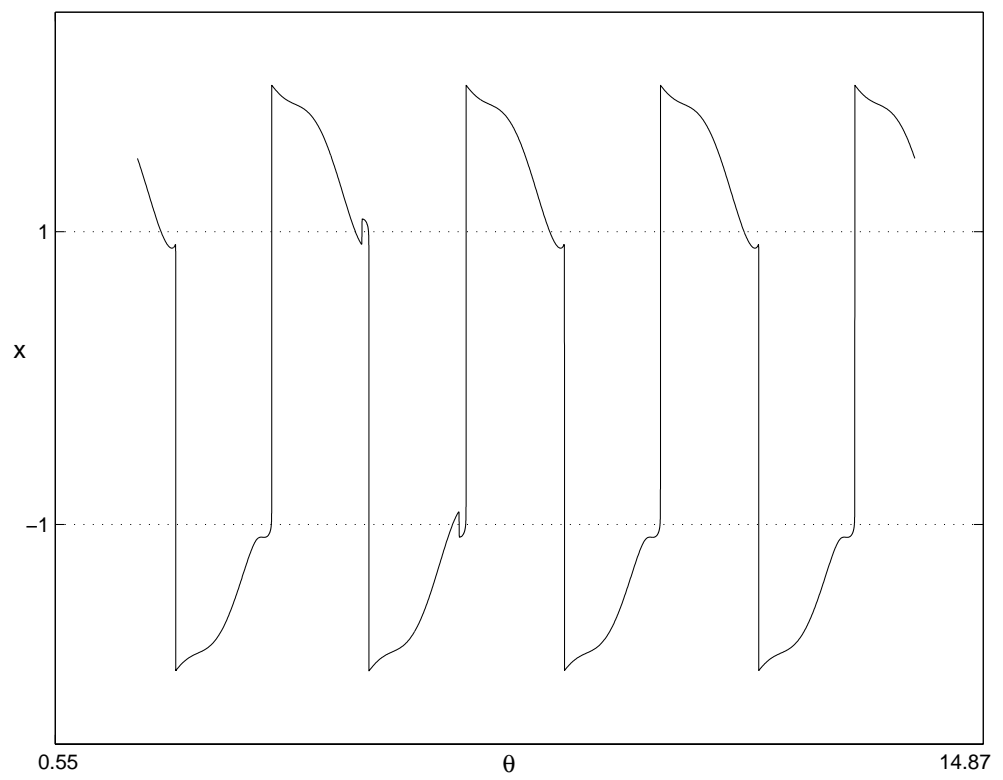

Figure 27. A graph of $x$ versus $\theta$ for a periodic orbit computed by AUTO. This solution is from the curve labeled "12b:anbbanan" in Figure 30. The symbol sequence anbbanan describes the behavior of the periodic orbit near the folded saddles. The parameter values are $\varepsilon=10^{-4}, a=1.1$, and $\omega=1.55$.

the singular nature of the limit $\varepsilon=0$. For example, homoclinic bifurcations or bifurcations associated with maximal canards in the reduced system are limits of infinite sets of bifurcations in (1.1) that collapse into one another as $\varepsilon \rightarrow 0$. Asymptotic analysis that is not pursued in this paper can be used to predict which bifurcations of periodic orbits of (1.1) will be found close to the bifurcations of the reduced system. Here we present several numerical examples that illustrate relationships between the bifurcations in (1.1) and the reduced system. The data presented here can be compared directly with predictions from asymptotic analysis.

Figure 27 shows a plot of $x$ versus $t$ along a periodic orbit of period 12. (Throughout the remainder of this section, the period of orbits is measured by $\theta$ rather than time. Thus a periodic orbit of period $k$ has winding number $k$ in the phase space.) This periodic orbit has five canards that can be identified as segments of the plot with $x \in(-1,1)$ and moderate slope. There are also three jumps without canards where $x$ crosses the entire interval $(-1,1)$ with very large slope. The jump back canards occur when the graph enters the region $|x|<1$ for a short distance but then has a short steep segment that leaves this region without crossing it. This is followed by a jump across $|x|<1$. In contrast, at the jump away canards, the trajectory enters the region $|x|<1$ along a segment with moderate slope and then crosses the region along a segment of very steep slope. The order in which the jumps occur is anbbanan where the symbol $a$ labels a jump away canard, the symbol $b$ labels a jump back canard, and the symbol $n$ labels a jump without canards.

Recall that the parameters $(a, \omega)=(1.1,1.55)$ lie in the region where the reduced system has a chaotic invariant set that is somewhere between a shift on two symbols and a shift on three symbols. The symbols are directly related to the $a, b, n$ we used to describe the jumps of 


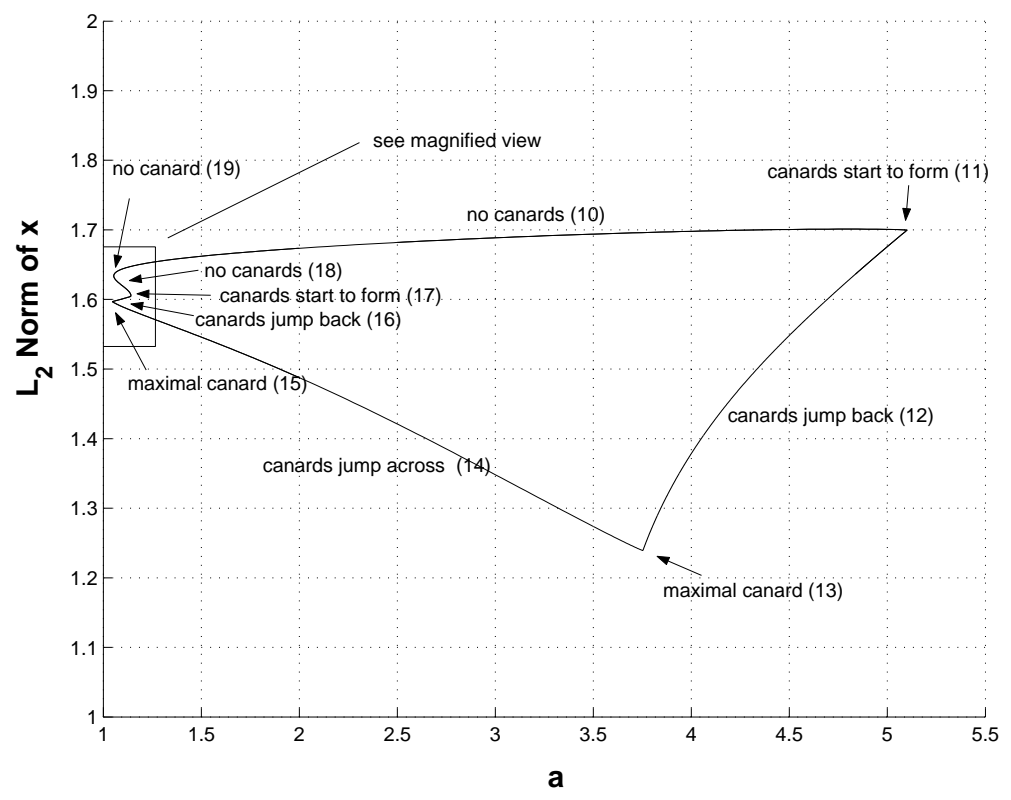

Figure 28. Symmetric periodic orbits of period 3 computed by AUTO. In this example, $\varepsilon=10^{-4}$ and $\omega=1.55$.

the periodic orbit in Figure 27. The subshift allows arbitrary sequences of these symbols with the single restriction that there is a limit on the length of consecutive $n$ 's. Thus the periodic orbit is compatible with the structure predicted by analysis of the reduced system and can be identified with a specific orbit in the nonwandering set of its extended half return map.

Figures 28, 29, and 30 show bifurcation diagrams for a few periodic orbits of periods 3 , 6 , and 12 (in $\theta$ ) computed by AUTO for system (1.1) with $\omega=1.55$ and $\varepsilon=10^{-4}$. The plots show the $L_{2}$ norm of $x$ along the orbit (normalized by its period) as a function of the continuation parameter $a$ in these calculations. The numbers in parentheses in Figures 28 and 29 are labels attached by AUTO to these solutions. We look at the dynamics of several below. The labels of each solution branch in Figure 30 identify the period and the sequence of jumps displayed by the orbits. In addition, the period 3 orbits are labeled with $\mathrm{A}$ for antisymmetric and $\mathrm{S}$ for symmetric. The fixed points of the extended half return map $G$ in this region correspond to symmetric periodic orbits of period 3. Asymmetric periodic orbits of period 3 correspond to periodic orbits of $G$ with period 2 .

We compare the data produced by these calculations with our analysis of bifurcations of $G$. Consider the bifurcations of circuit number 3 fixed points encountered along the line $\omega=1.55$ in Figure 22. The right-hand boundary of the region with circuit number 3 fixed points is a curve of homoclinic orbits. The point labeled (11) in Figure 28 occurs where there is an apparent saddle-node bifurcation. This saddle-node bifurcation is related to the homoclinic bifurcation of the reduced system. The most direct analogue of a homoclinic bifurcation of the reduced system in the three dimensional flow consists of periodic orbits that have a tangency with the surfaces $x= \pm 1$. (Since the orbits are symmetric, there will be tangencies with both $x=1$ and $x=1$.) Figure 31 shows a plot of $x$ versus $t$ for the orbit labeled (11). This orbit 


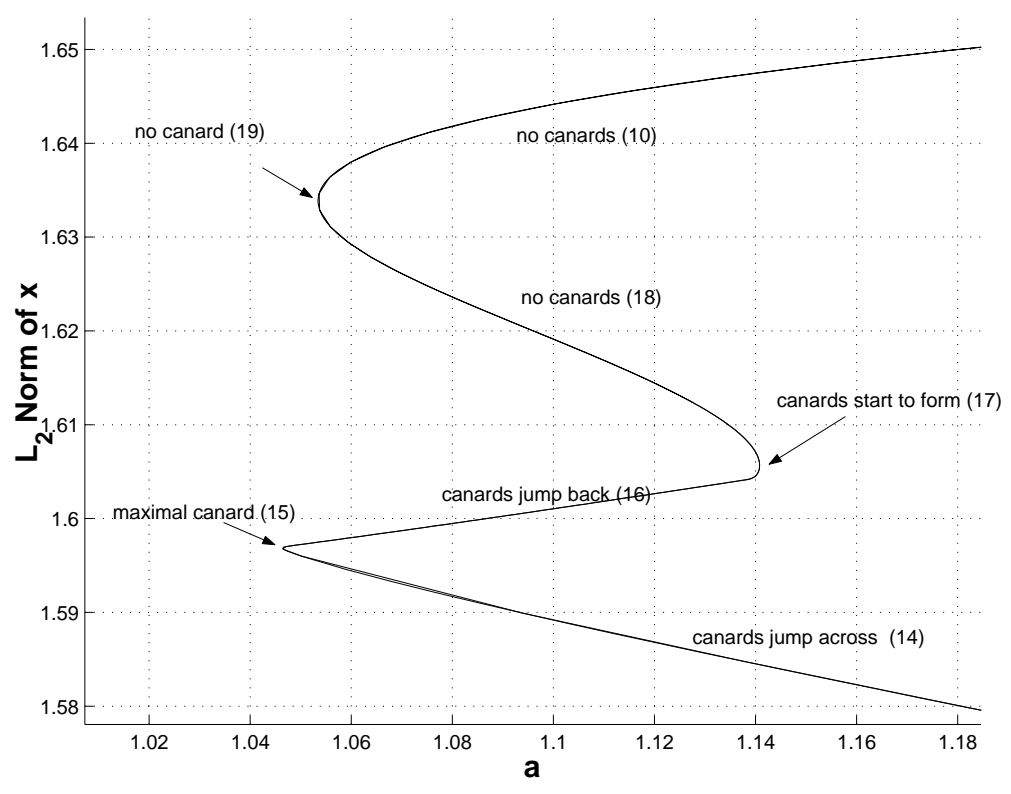

Figure 29. A magnified view of the boxed region in Figure 28.

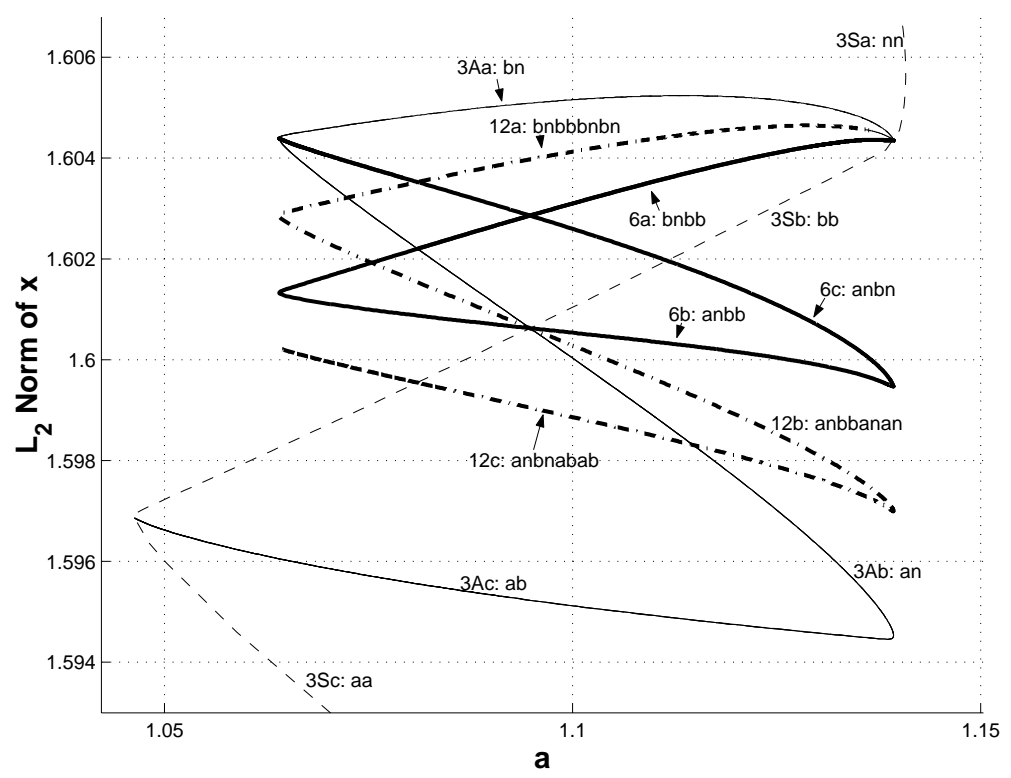

Figure 30. A partial bifurcation diagram of periodic orbits computed by AUTO. In this example, $\varepsilon=10^{-4}$ and $\omega=1.55$.

appears to be very close to one with a tangency with the surfaces $x= \pm 1$. We conjecture that the relationship between a homoclinic bifurcation of the reduced system and the presence of saddle-node bifurcations in the "full" system is a general one [13]. We also note that the apparent vertex of the bifurcation curve at the point labeled (11) seems to be a smooth fold 


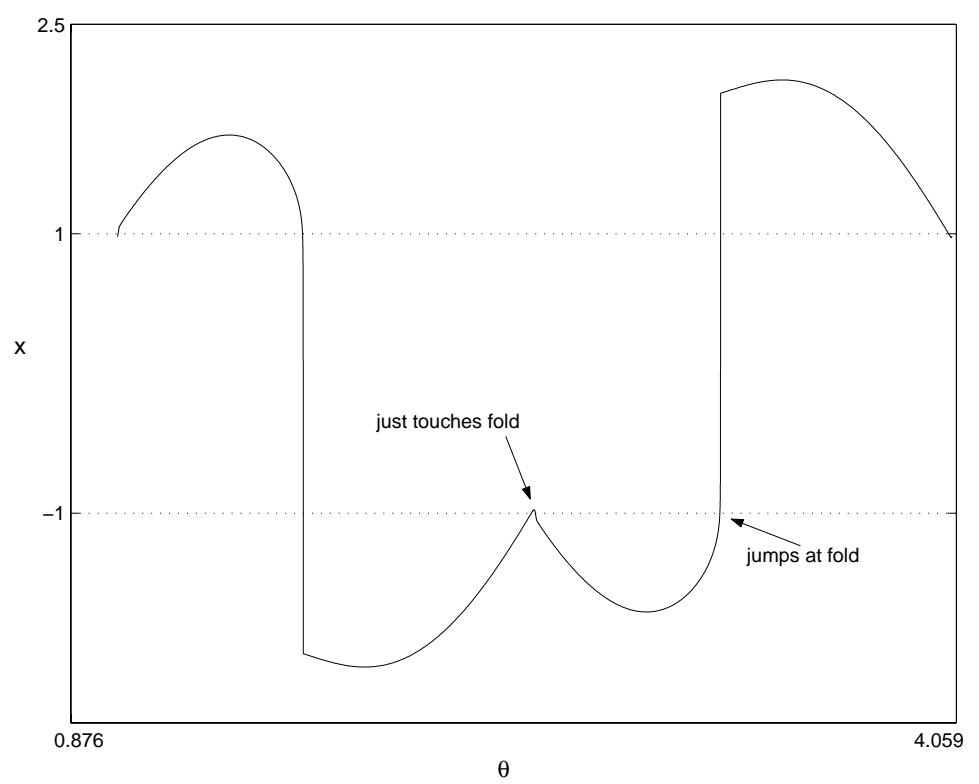

Figure 31. The periodic orbit labeled (11) in Figure 28. A jump back canard has just begun to form.

in a blowup of the region near this turning point in Figure 28.

Decreasing $a$, the next bifurcation curve of fixed points with circuit number 3 that we encounter is the red curve at which $G$ has a maximal canard fixed point. This corresponds to the point labeled (13) in Figure 28. The periodic orbit is plotted in Figure 32. In this figure, observe that the periodic orbit crosses the surface $x=-1$ without jumping, returning to the surface $x=-1$ before finally jumping. This portion of the periodic orbit is the maximal canard. There is no bifurcation of the family of periodic orbits in this region, but the type of canard contained in the orbit switches from a jump back canard to a jump across canard.

Further bifurcations of the symmetric periodic orbits are best viewed in Figures 14 and 29, which show the regions with finer resolution. The next two bifurcations encountered in the reduced system are very close together. Figure 14 shows that a saddle-node bifurcation curve is crossed (with decreasing a) shortly before a homoclinic bifurcation curve is crossed. In Figure 29, we see a fold at the point labeled (17) near $a=1.14$ that occurs just before canards begin to form. The homoclinic bifurcation is on the boundary of the region in which $G$ has a chaotic invariant set. For the first time, we find parameters at which there are two periodic orbits with canards. Figure 33 shows an example of a periodic orbit with jump back canards that appear as $a$ is decreased further. There is no apparent bifurcation of the periodic orbits themselves associated with this crossing of the homoclinic curve, but we see below that there are bifurcations in this region giving rise to additional periodic orbits that presumably lie in a chaotic invariant set.

There are two additional bifurcations that we see on the family of periodic orbits. The first of these is another saddle-node bifurcation of a trajectory with no canards. The saddlenode bifurcations of $G$ are located along the green curve in Figure 14. The last bifurcation of the family of periodic orbits occurs where there is a maximal canard. Figure 34 shows this 


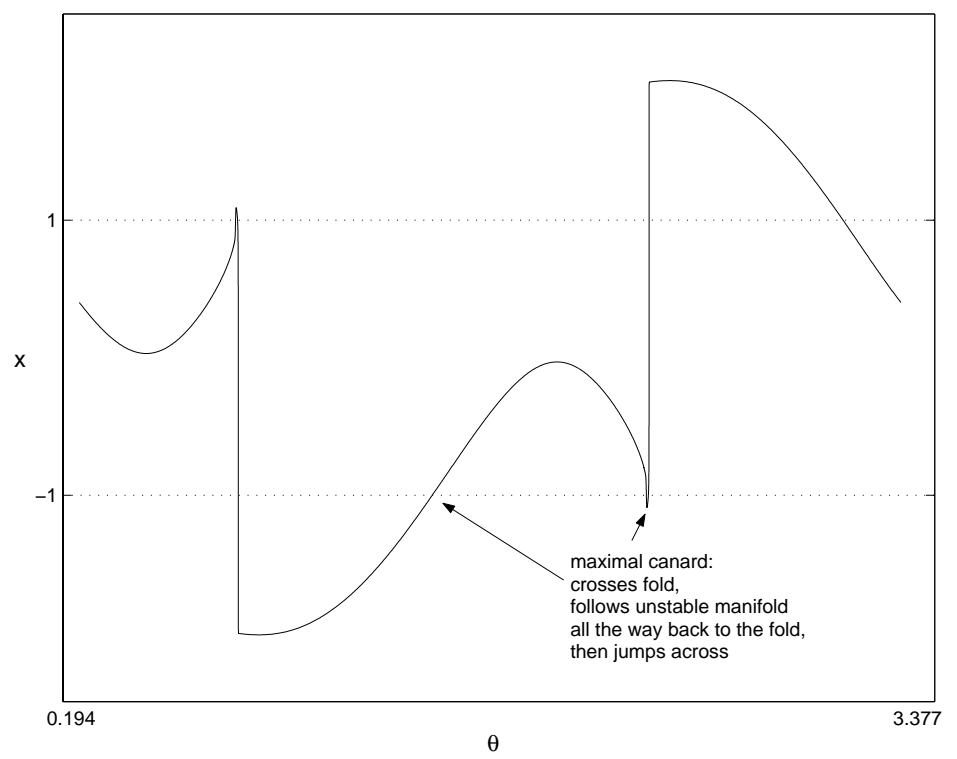

Figure 32. The periodic orbit labeled (13) in Figure 28. There is a maximal canard in which the trajectory crosses the surface $x=-1$ and then returns to this surface before jumping.

trajectory. The trajectory crosses the fold curve and continues along the slow manifold until it returns to the fold. There it jumps. This bifurcation corresponds to the maximal canard fixed point curve drawn in red in Figure 14. Here $\theta_{m}=\theta_{2 s}$ is a fixed point for $G$. This is the boundary of the region where $G$ has a chaotic invariant set. For smaller values of $a, G$ has no canard fixed points.

Let us turn now to Figure 30. The dashed curve is an expanded view of the portion of the curve of symmetric periodic orbits displayed in Figure 29 that contains jump back canards and the point labeled (16). This is the region where we expect to see chaotic invariant sets, including the additional periodic orbits displayed in Figure 30. In the region in which $G$ has a chaotic invariant topologically equivalent to a three shift, there are three fixed points. These correspond to the symmetric periodic orbits in the diagram, with symbol sequences $a a, b b$, and $n n$ for the full return map. There are also three asymmetric periodic orbits with symbol sequences $a b$, an, and $b n$ corresponding to the three periodic orbits of period 2 in the shift on three symbols. The diagram also shows a branch of period 6 and a branch of period 12 orbits, each of which has two turning points.

It is notable that many turning points of the branches seem to occur at almost the same value of the continuation parameter $a$. In generic dynamical systems, there is no reason that several bifurcations should happen simultaneously. However, the very close proximity of these bifurcations to one another is a consequence of the strong contraction and expansion associated with the two time scales in the van der Pol system. The right-hand bifurcation of these branches occurs very close to the homoclinic bifurcation curve that bounds the region where $G$ has a chaotic invariant set. In the van der Pol system, we expect a cascade of bifurcations to occur in a small parameter interval whose length shrinks rapidly to 0 as $\varepsilon \rightarrow 0$. 


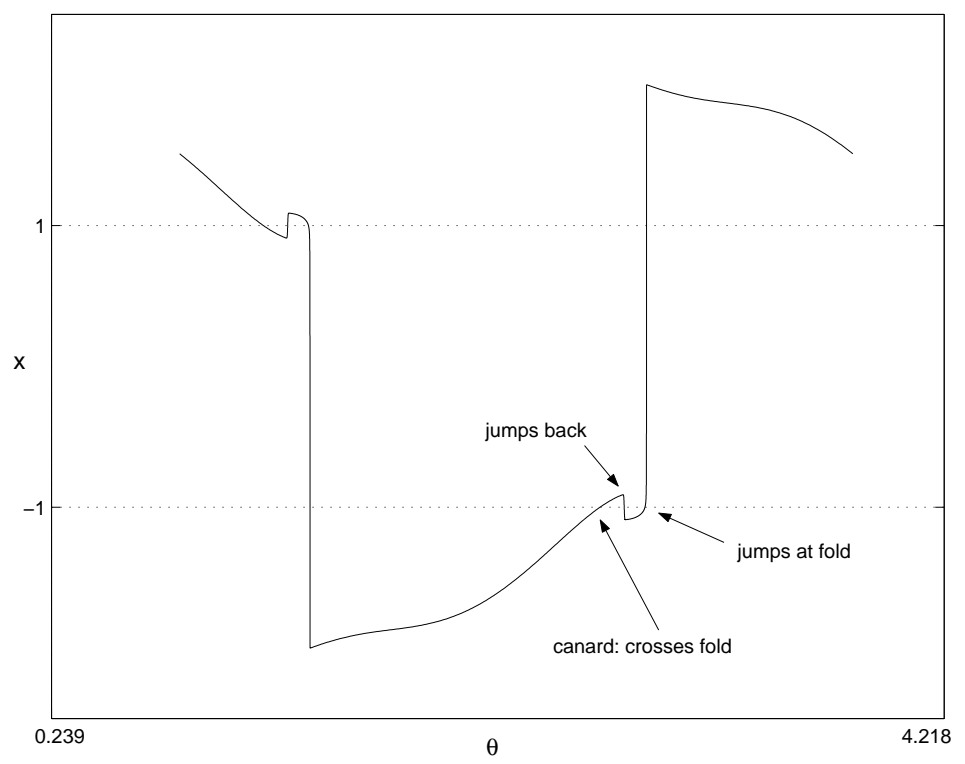

Figure 33. The periodic orbit labeled (16) in Figure 28. A jump back canard has just begun to form.

This explains the location of the right-hand turning points for the asymmetric branches shown in Figure 30. The left-hand turning points seem to occur together at a value of the parameter $a$ where there are no bifurcations of the symmetric period 3 orbits. However, note that all of the branches that are displayed contain a jump without canards. This means that these periodic orbits come from invariant sets that are larger than a two shift. In Figure 14, the yellow curve displays bifurcations in which $G^{2}$ maps the maximal canard point back to the discontinuity of $G$. In section 5 we showed that, to the left of this curve, the chaotic invariant set of $G$ is topologically equivalent to a two shift and consists entirely of $G$ trajectories within the canard extensions of $H$. Thus we conclude that the left-hand turning points of the asymmetric orbits in Figure 30 correspond to this bifurcation of $G$.

We make a final observation about Figure 30 that is not related to bifurcations. It appears that all four branches of periodic orbits pass through the same point in the middle of the diagram. In fact, the curves are only close to having a common intersection. The presence of this close intersection is readily explained. The period 6 and period 12 orbits are formed from segments that are almost concatenations of the symmetric and asymmetric period 3 orbits. Therefore, where the two period 3 orbits have the same $L_{2}$ norm, the period 6 and period 12 orbits have almost the same norm.

Through these examples, we have shown that there is a very close qualitative correspondence between the bifurcations of the extended half return map $G$ and the bifurcations of periodic orbits in the van der Pol system for $\varepsilon=10^{-4}$. The location of bifurcation curves in the $(a, \omega)$ plane has shifted slightly and there is a translation between some types of bifurcations, but the numerical results illustrate that analysis of the reduced system gives a good strategy for the van der Pol system. 


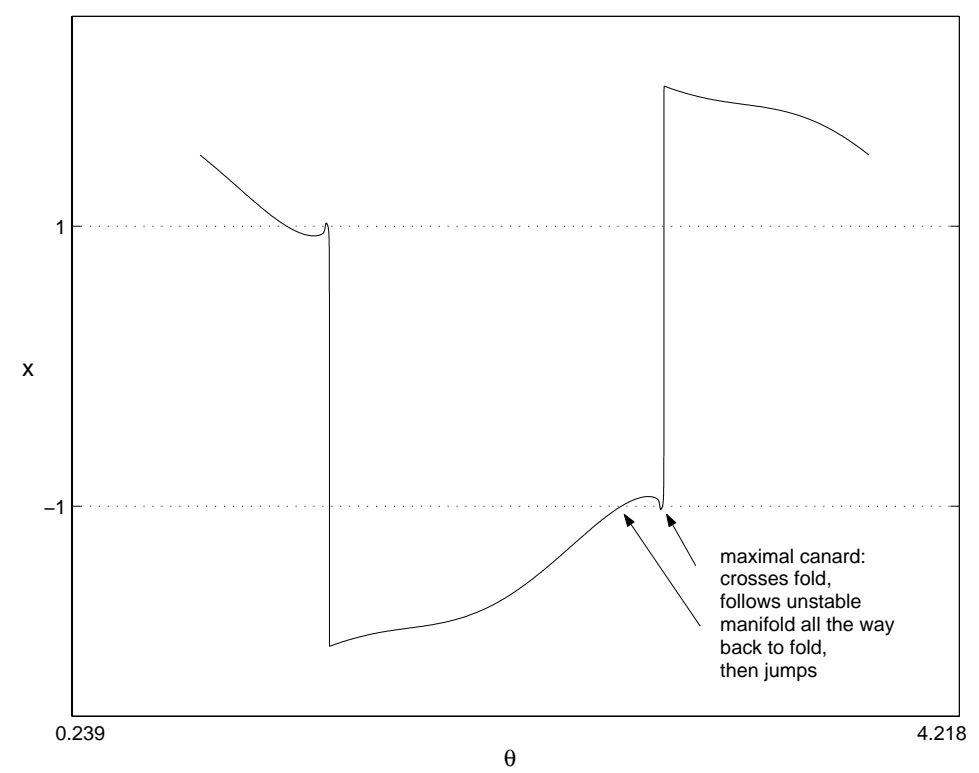

Figure 34. The periodic orbit labeled (15) in Figure 28. There is a maximal canard that is much shorter than the maximal canard shown in Figure 32.

8. Concluding remarks. The ultimate goal of this research is to describe the dynamics and bifurcations of the forced van der Pol vector field (1.1). This paper is a step toward that goal, but there is much that remains to be done. We have demonstrated how to extend the half return map of the reduced system to approximate the location of trajectories with canards in the van der Pol system. Furthermore, we have shown how symbolic dynamics can be used to characterize the dynamics on chaotic invariant sets. Each point in the canard extensions of $H$ corresponds to a uniquely determined canard of the reduced system. The trajectories of the reduced system are limits of trajectory families of the van der Pol system (1.1) as $\varepsilon \rightarrow 0$. This limit is highly singular, and the analysis here hardly touches upon the nature of this approximation. Instead, we present numerical studies that illustrate the relationship between the two systems.

We have also shown how methods used to analyze bifurcations of one dimensional maps can be applied to the extended half return map $G$. We have computed curves in parameter space of several types of bifurcations that involve canards. Bifurcations of the van der Pol system (1.1) appear close to the bifurcations of the reduced system that we have identified, but some have a different character. For example, at points where two coincident maximal canard points are fixed, the van der Pol system will have behavior reminiscent of the bifurcations displayed by the Henon mapping $[3,16,25]$. Instead of the immediate creation of a chaotic invariant set, we believe that there is an "exponentially thin" region of trajectories that contains maximal canards. "Normal forms" for maximal canards of a slow-fast system can be derived from the analysis of Arnold et al. [1], but the role that these trajectories play in the global analysis of relaxation oscillations has not been considered. We conjecture that the return map of slowfast systems near maximal canards have folds that are similar to the Henon mapping with 
small positive Jacobian. We hope that systematic investigation with asymptotic methods of the codimension one degenerate slow-fast decompositions in the van der Pol vector field and its reduced system will enable the relationship between these two systems to be made more precise.

\section{REFERENCES}

[1] V. I. Arnold, V. S. Afrajmovich, Yu. S. Il'yashenko, and L. P. Shil'nikov, Dynamical Systems V, Encyclopaedia Math. Sci. 5, Springer-Verlag, Berlin, 1994.

[2] A. Back, J. Guckenheimer, And M. Myers, A dynamical simulation facility for hybrid systems, in Hybrid Systems, Lecture Notes in Comput. Sci. 736, Springer-Verlag, Berlin, 1993, pp. 255-267.

[3] M. Benedicks and L. Carleson, The dynamics of the Henon map, Ann. of Math. (2), 133 (1991), pp. 73-169.

[4] É. Benô̂t, Canards et enlacements, Inst. Hautes Études Sci. Publ. Math., 72 (1990), pp. 63-91.

[5] L. Block And J. Franke, Existence of periodic points for maps of $S^{1}$, Invent. Math., 22 (1973/1974), pp. 69-73.

[6] M. Cartwright and J. Littlewood, On nonlinear differential equations of the second order II: The equation $\ddot{y}-k f(y, \dot{y}) \dot{y}+g(y, k)=p(t)=p_{1}(t)+k p_{2}(t), k>0, f(y) \geq 1$, Ann. of Math. (2), 48 (1947), pp. 472-494.

[7] M. Cartwright and J. Littlewood, Addendum to "On nonlinear differential equations of the second order II," Ann. of Math. (2), 50 (1949), pp. 504-505.

[8] W. De Melo and S. van Strien, One-Dimensional Dynamics, Ergebnisse der Mathematik und ihrer Grenzgebiete (3) [Results in Mathematics and Related Areas (3)] 25, Springer-Verlag, Berlin, 1993.

[9] E. Doedel, R. Paffenroth, A. R. Champneys, T. F. Fairgrieve, Y. A. Kuznetsov, B. SandSTEDE, AND X. WANG, AUTO 2000: Continuation and Bifurcation Software for Ordinary Differential Equations (with HomCont), Technical report, California Institute of Technology, Pasadena, CA, 2001.

[10] N. Fenichel, Persistence and smoothness of invariant manifolds for flows, Indiana Univ. Math. J., 21 (1971), pp. 193-225.

[11] J. Guckenheimer, On the bifurcation of maps of the interval, Invent. Math., 39 (1977), pp. 165-178.

[12] J. Guckenheimer, Sensitive dependence to initial conditions for one dimensional maps, Comm. Math. Phys., 70 (1979), pp. 133-160.

[13] J. Guckenheimer, Bifurcation and degenerate decomposition in multiple time scale dynamical systems, in Nonlinear Dynamics and Chaos: Where Do We Go from Here?, J. Hogan, A. Champneys, B. Krauskopf, M. di Bernardo, E. Wilson, H. Osinga, and M. Homer, eds., Institut of Physics Publishing, Bristol, UK, 2002, pp. 1-21.

[14] J. Guckenheimer, K. Hoffman, And W. Weckesser, The forced van der Pol equation I: The slow flow and its bifurcations, SIAM J. Appl. Dyn. Syst., 2 (2003), pp. 1-35.

[15] J. GuCKenheimer And B. Meloon, Computing periodic orbits and their bifurcations with automatic differentiation, SIAM J. Sci. Comput., 22 (2000), pp. 951-985.

[16] M. Henon, A two-dimensional mapping with a strange attractor, Comm. Math. Phys., 50 (1976), pp. 69-77.

[17] N. Levinson, Perturbations of discontinuous solutions of non-linear systems of differential equations, Acta Math., 82 (1950), pp. 71-106.

[18] J. LitTlewood, On nonlinear differential equations of the second order III: The equation $\ddot{y}-k\left(1-y^{2}\right) \dot{y}+$ $y=b k \cos (\lambda t+a)$ for large $k$ and its generalizations, Acta Math., 97 (1957), pp. 267-308. (Errata, Acta Math., 98 (1957), p. 110.)

[19] J. LitTlewood, On nonlinear differential equations of the second order IV: The general equation $\ddot{y}-$ $k f(y) \dot{y}+g(y)=b k p(\phi), \phi=t+a$ for large $k$ and its generalizations, Acta Math., 98 (1957), pp. $1-110$.

[20] J. Milnor And W. Thurston, On iterated maps of the interval, in Dynamical Systems (College Park, MD, 1986-1987), Lecture Notes in Math. 1342, Springer-Verlag, Berlin, 1988, pp. 465-563. 
[21] E. Mishchenko, Yu. Kolesov, A. Kolesov, and N. Rozov, Asymptotic Methods in Singularly Perturbed Systems, Translated from the Russian by Irene Aleksanova, Monogr. Comtemp. Math., Consultants Bureau, New York, 1994.

[22] S. Smale, Differentiable dynamical systems, Bull. Amer. Math. Soc., 73 (1967), pp. 747-817.

[23] P. Szmolyan and M. Wechselberger, Canards in $R^{3}$, J. Differential Equations, 177 (2001), pp. 419453.

[24] B. VAN DER POL, The nonlinear theory of electric oscillations, Proc. IRE, 22 (1934), pp. 1051-1086.

[25] Q. Wang And L.-S. Young, From invariant curves to strange attractors, Comm. Math. Phys., 225 (2002), pp. 275-304. 